\title{
Mesons with beauty and charm: New horizons in spectroscopy
}

\author{
Estia J. Eichten ${ }^{*}$ and Chris Quigg ${ }^{\dagger}$ \\ Fermi National Accelerator Laboratory, P.O. Box 500, Batavia, Illinois 60510, USA
}

(Received 8 March 2019; published 28 March 2019)

\begin{abstract}
The $B_{c}^{+}$family of $(c \bar{b})$ mesons with beauty and charm is of special interest among heavy quarkonium systems. The $B_{c}^{+}$mesons are intermediate between $(c \bar{c})$ and $(b \bar{b})$ states both in mass and size, so many features of the $(c \bar{b})$ spectrum can be inferred from what we know of the charmonium and bottomonium systems. The unequal quark masses mean that the dynamics may be richer than a simple interpolation would imply, in part because the charmed quark moves faster in $B_{c}$ than in the $J / \psi$. Close examination of the $B_{c}^{+}$spectrum can test our understanding of the interactions between heavy quarks and antiquarks and may reveal where approximations break down. Whereas the $J / \psi$ and $\Upsilon$ levels that lie below the flavor threshold are metastable with respect to strong decays, the $B_{c}$ ground state is absolutely stable against strong or electromagnetic decays. Its dominant weak decays arise from $\bar{b} \rightarrow \bar{c} W^{\star+}, c \rightarrow s W^{\star+}$, and $c \bar{b} \rightarrow W^{\star+}$ transitions, where $W^{\star}$ designates a virtual weak boson. Prominent examples of the first category are quarkonium transmutations such as $B_{c}^{+} \rightarrow J / \psi \pi^{+}$and $B_{c}^{+} \rightarrow J / \psi \ell^{+} \nu_{\ell}$, where $J / \psi$ designates the $(c \bar{c}) 1 S$ level. The high data rates and extraordinarily capable detectors at the Large Hadron Collider give renewed impetus to the study of mesons with beauty and charm. Motivated by the recent experimental searches for the radially excited $B_{c}$ states, we update the expectations for the low-lying spectrum of the $B_{c}$ system. We make use of lattice QCD results, a novel treatment of spin splittings, and an improved quarkonium potential to obtain detailed predictions for masses and decays. We suggest promising modes in which to observe excited states at the LHC. The $3 P$ and $3 S$ states, which lie close to or just above the threshold for strong decays, may provide new insights into the mixing between quarkonium bound states and nearby two-body open-flavor channels. Searches in the $B^{(*)} D^{(*)}$ final states could well reveal narrow resonances in the $J^{P}=0^{-}, 1^{-}$, and $2^{+}$channels and possibly in the $J^{P}=0^{+}$and $1^{+}$channels at threshold. Looking further ahead, the prospect of very-high-luminosity $e^{+} e^{-}$colliders capable of producing tera- $Z$ samples raises the possibility of investigating $B_{c}$ spectroscopy and rare decays in a controlled environment.
\end{abstract}

DOI: 10.1103/PhysRevD.99.054025

\section{INTRODUCTION}

Although the lowest-lying $(c \bar{b})$ meson has long been established, the spectrum of excited states is little explored. The ATLAS experiment at CERN's Large Hadron Collider reported the observation of a radially excited $B_{c}$ state [1], but this sighting was not confirmed by the LHC $b$ experiment [2]. The unsettled experimental situation and the large datasets now available for analysis make it timely for us to provide up-to-date theoretical expectations for the spectrum and decay patterns of narrow $(c \bar{b})$ states and for their production in hadron colliders [3]. New work from the CMS Collaboration [4]

\footnotetext{
eichten@fnal.gov

†uigg@fnal.gov
}

Published by the American Physical Society under the terms of the Creative Commons Attribution 4.0 International license. Further distribution of this work must maintain attribution to the author(s) and the published article's title, journal citation, and DOI. Funded by SCOAP ${ }^{3}$. shows the way toward exploiting the potential of $(c \bar{b})$ spectroscopy.

\section{A. What we know of the $B_{c}$ mesons}

The possibility of a spectrum of narrow $B_{c}$ states was first suggested by Eichten and Feinberg [5]. Anticipating the copious production of $b$-quarks at Fermilab's Tevatron Collider and CERN's Large Electron-Positron Collider (LEP), we presented a comprehensive portrait of the spectroscopy of the $B_{c}$ meson and its long-lived excited states [6], based on then-current knowledge of the interaction between heavy quarks derived from $(c \bar{c})$ and $(b \bar{b})$ bound states, within the framework of nonrelativistic quantum mechanics [7]. Surveying four representative potentials, we characterized the mass of the $J^{P}=0^{-}$ground state as $M\left(B_{c}\right) \approx 6258 \pm 20 \mathrm{MeV}$. A small number of $B_{c}$ candidates appeared in hadronic $Z^{0}$ decays at LEP. The CDF Collaboration observed the decay $B_{c}^{ \pm} \rightarrow J / \psi \ell^{ \pm} \nu$ in 1.8 $\mathrm{TeV} \bar{p} p$ collisions at the Fermilab Tevatron [8], estimating the mass as $M\left(B_{c}\right) \approx 6400 \pm 411 \mathrm{MeV}$. (The generic 
lepton $\ell$ represents an electron or muon.) Subsequent work by the CDF [9], D0 [10], and $\mathrm{LHC} b$ [11] Collaborations has refined the mass to $M\left(B_{c}\right)=6274.9 \pm 0.8 \mathrm{MeV}$ [12], with the most precise determinations coming from fully reconstructed final states such as $J / \psi \pi^{+}$.

Investigations based on the spacetime lattice formulation of QCD aim to provide $a b$ initio calculations that incorporate the full dynamical content of the theory of strong interactions. Before the nonleptonic $B_{c}$ decays had been observed, a first unquenched lattice QCD prediction, incorporating $2+1$ dynamical quark flavors $(u / d, s)$ found $M\left(B_{c}\right)=6304 \pm 12_{-0}^{+18} \mathrm{MeV}$ [13], where the first error bar represents statistical and systematic uncertainties and the second characterizes heavy-quark discretization effects. Calculations incorporating $2+1+1$ dynamical quark flavors $(u / d, s, c)$ [14] yield $M\left(1^{1} S_{0}\right)=6278 \pm 9 \mathrm{MeV}$, in impressive agreement with the measured $B_{c}$ mass, and predict $M\left(2^{1} S_{0}\right)=6894 \pm 19 \pm 8 \mathrm{MeV}$ [15].

Three distinct elementary processes contribute to the decay of $B_{c}$ : the individual decays $\bar{b} \rightarrow \bar{c} W^{\star+}$ and $c \rightarrow$ $s W^{\star+}$ of the two heavy constituents, and the annihilation $c \bar{b} \rightarrow W^{\star+}$ through a virtual $W$-boson. Several examples of the $\bar{b} \rightarrow \bar{c}$ transition have been observed, including the final states $J / \psi \ell^{+} \nu_{\ell}, J / \psi \pi^{+}, J / \psi K^{+}, J / \psi \pi^{+} \pi^{+} \pi^{-}$, $J / \psi \pi^{+} K^{+} K^{-}, J / \psi \pi^{+} \pi^{+} \pi^{+} \pi^{-} \pi^{-}, J / \psi D_{s}^{+}, J / \psi D_{s}^{\star+}$, and $J / \psi \pi^{+} \bar{p} p$; and $\psi(2 S) \pi^{+}$. A single channel, $B_{s}^{0} \pi^{+}$, representing the $c \rightarrow s$ transition is known. The annihilation mechanism, which would lead to final states such as $\tau^{+} \nu_{\tau}$ and $\bar{p} p \pi^{+}$, has not yet been established. The observed lifetime, $\tau\left(B_{c}\right)=(0.507 \pm 0.009)$ ps [12], is consistent with theoretical expectations $[16,17]$. Predictions for partial decay rates (or relative branching fractions) await experimental tests. Some recent theoretical works explore the potential of rare $B_{c}$ decays [18].

Until recently, the only evidence reported for a $(c \bar{b})$ excited state was presented by the ATLAS Collaboration [1] in $p p$ collisions at 7 and $8 \mathrm{TeV}$, in samples of 4.9 and $19.2 \mathrm{fb}^{-1}$. They observed a new state at $6842 \pm 7 \mathrm{MeV}$ in the $M\left(B_{c}^{ \pm} \pi^{+} \pi^{-}\right)-M\left(B_{c}^{ \pm}\right)-2 M\left(\pi^{ \pm}\right)$mass difference, with $B_{c}^{ \pm}$detected in the $J / \psi \pi^{ \pm}$mode. The mass $(527 \pm 7 \mathrm{MeV}$ above $\langle M(1 S)\rangle)$ and decay of this state are broadly in line with expectations for the second $s$-wave state, $B_{c}^{ \pm}(2 S)$. In addition to the nonrelativistic potentialmodel calculations cited above, the HPQCD Collaboration has presented preliminary results from a lattice calculation using $2+1+1$ dynamical fermion flavors and highly improved staggered quark correlators [19]. They report $M\left(2^{1} S_{0}\right)=6892 \pm 41 \mathrm{MeV}$, which is $576.5 \pm 41 \mathrm{MeV}$ above $\langle M(1 S)\rangle)$. This result and the NRQCD prediction [14] lie above the ATLAS report by 1 and 2 standard deviations, respectively. The significance of the discrepancy is limited for the moment by lattice uncertainties. A plausible interpretation has been that ATLAS might have observed the transition $B_{c}^{*}(2 S) \rightarrow B_{c}^{*}(1 S) \pi^{+} \pi^{-}$, missing the low-energy photon from the subsequent $B_{c}^{*} \rightarrow B_{c} \gamma$ decay, and that the signal is an unresolved combination of $2^{3} S_{1}$ and $2^{1} S_{0}$ peaks. A search by the LHC $b$ collaboration in $2 \mathrm{fb}^{-1}$ of 8 -TeV $p p$ data yielded no evidence for either $B_{c}(2 S)$ state [2]. As we prepared this article for publication, the CMS Collaboration provided striking evidence for both $B_{c}(2 S)$ levels, in the form of wellseparated peaks in the $B_{c} \pi^{+} \pi^{-}$invariant mass distribution, closely matching the theoretical template [4]. We incorporate these new observations into the discussion that follows in Sec. VA.

\section{B. Analyzing the $(c \bar{b})$ bound states}

The nonrelativistic potential picture, motivated by the asymptotic freedom of QCD [20], gave early insight into the nature of charmonium and generated a template for the spectrum of excited states [21]. For more than four decades, it has served as a reliable guide to quarkonium spectroscopy, including the states lying near or just above flavor threshold for fission into two heavy-light mesons that are significantly influenced by coupled-channel effects [22,23].

We view the nonrelativistic potential-model treatment as a stepping stone, not a final answer, however impressive its record of utility. Potential theory does not capture the full dynamics of the strong interaction, and while the standard coupled-channel treatment is built on a plausible physical picture, it is not derived from first principles. Moreover, relativistic effects may be more important for $(c \bar{b})$ than for $(c \bar{c})$. The $c$-quark moves faster in the $B_{c}$ meson than in the $J / \psi$, because it must balance the momentum of a more massive $b$-quark. One developing area of theoretical research has been to explore methods more robust than nonrelativistic quantum mechanics [17,24,25].

Nonperturbative calculations on a spacetime lattice in principle embody the full content of QCD. This approach is yielding increasingly precise predictions for the masses of $(c \bar{b})$ levels up through the $B_{c}^{* \prime}\left(2^{3} S_{1}\right)$ state. It is not yet possible to extract reliable signals for higher-lying states from the lattice, so we rely on potential-model methods to construct a template for the $B_{c}$ spectrum through the $4^{3} S_{1}$ level. If experiments should uncover systematic deviations from the expectations we present, they may be taken as evidence of dynamical features absent from the nonrelativistic potential-model paradigm, including - of coursecoupling to states above flavor threshold, which we neglect in our calculations of the spectrum.

In the following Sec. II, we develop the theoretical tools required to compute the $(c \bar{b})$ spectrum. In earlier work [6], we examined the Cornell Coulomb-plus-linear potential [22], a power-law potential [26], Richardson's QCDinspired potential [27], and a second QCD-inspired potential due to Buchmüller and Tye [28], which we took as our reference model. We used a perturbation-theory treatment of spin splittings. Using insights from lattice QCD and 
higher-order perturbative calculations, we construct a new potential that differs in detail from those explored in earlier work. We also use lattice results and rich experimental information on the $(c \bar{c})$ and $(b \bar{b})$ spectra to refine the treatment of spin splittings. We present our expectations for the spectrum of narrow states in Sec. III. We consider decays of the narrow states in Sec. IV, updating the results we gave in Ref. [6]. We compute differential and integrated cross sections for the narrow $B_{c}$ levels in proton-proton collisions at the Large Hadron Collider in Sec. V. Putting all these elements together, we show how to unravel the $2 S$ levels and explore how higher levels might be observed. Prospects for a future $e^{+} e^{-} \rightarrow$ tera-Z machine appear in Sec. VI. We draw some conclusions and look ahead in Sec. VII.

\section{THEORETICAL PRELIMINARIES}

We take as our starting point a Coulomb-plus-linear potential (the "Cornell potential" [22]),

$$
V(r)=-\frac{\kappa}{r}+\frac{r}{a^{2}},
$$

where $\kappa \equiv 4 \alpha_{\mathrm{s}} / 3=0.52$ and $a=2.34 \mathrm{GeV}^{-1}$ were chosen to fit the quarkonium spectra. Analysis of the $J / \psi$ and $\Upsilon$ families led to the choices

$$
m_{c}=1.84 \mathrm{GeV} \quad m_{b}=5.18 \mathrm{GeV} .
$$

This simple form has been modified to incorporate running of the strong coupling constant in Refs. [27,28], among others, using the perturbative-QCD evolution equation at leading order and beyond. At distances relevant for confinement, perturbation theory ceases to be a reliable guide. It is now widely held, following Gribov [29], that as a result of quantum screening $\alpha_{\mathrm{s}}$ approaches a critical, or frozen, value at long distances (low-energy scales). In a light $(q \bar{q})$ system, Gribov estimated

$$
\alpha_{\mathrm{s}} \rightarrow \bar{\alpha}_{\mathrm{s}}=\frac{3 \pi}{4}(1-\sqrt{2 / 3}) \approx 0.14 \pi=0.44 .
$$

We incorporate the spirit of this insight into a new version of the Coulomb-plus-linear form that we call the "frozen- $\alpha_{\mathrm{s}}$ potential."

The long-range part is the standard Cornell linear term. To obtain the Coulomb piece, we convert the four-loop running of $\alpha_{\mathrm{s}}(q)$ in momentum space [30] to the behavior in position space using the method of [31], with an important modification. We set $\alpha_{\mathrm{s}}(q=1.6 \mathrm{GeV})=0.338$ and evolve with three active quark flavors. To enforce saturation of $\alpha_{\mathrm{S}}(r)$ at long distances, we alter the recipe of Ref. [31], replacing the identification $q=1 / r \exp \left(\gamma_{\mathrm{E}}\right)$, where $\gamma_{\mathrm{E}}=$ $0.57721 \ldots$ is Euler's constant, with the damped form $q=1 /\left[\left(r \exp \left(\gamma_{\mathrm{E}}\right)^{2}+\mu^{2}\right]^{1 / 2}\right.$. For our reference potential, we have chosen the damping parameter $\mu=1.2 \mathrm{GeV}$.

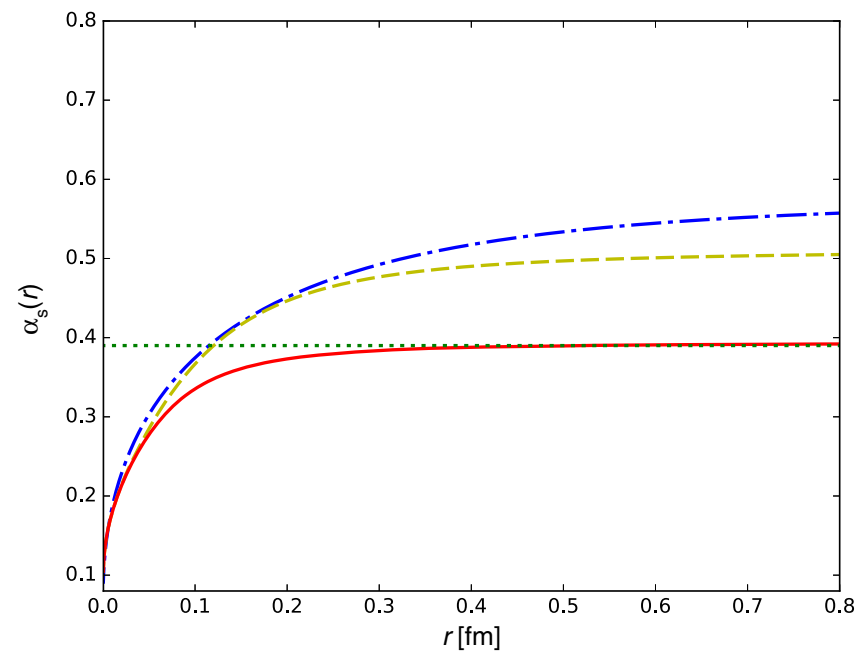

FIG. 1. Dependence of the running coupling $\alpha_{\mathrm{s}}(r)$ on the interquark separation $r$. The strong coupling for our chosen potential is shown in the solid red curve. Those corresponding to the Cornell potential (green dots) [22], Richardson potential (blue dash-dotted) [27], and an alternative version of the new potential with $\mu=0.8 \mathrm{GeV}$ (gold dashes) are shown for comparison.

The consequent evolution of $\alpha_{\mathrm{s}}(r)$ is plotted as the solid red curve in Fig. 1, where we also show an alternative choice of $\mu=0.8 \mathrm{GeV}$ (dashed gold curve), the constant $\alpha_{\mathrm{s}}$ of the original Cornell potential (dotted green curve), and $\alpha_{\mathrm{s}}(r)$ corresponding to the Richardson potential (dot-dashed blue curve).

We plot in Fig. 2 the frozen- $\alpha_{\mathrm{s}}$ potential for both our chosen example, $\mu=1.2 \mathrm{GeV}$, and the alternative, $\mu=0.8 \mathrm{GeV}$. There we also show the Richardson and

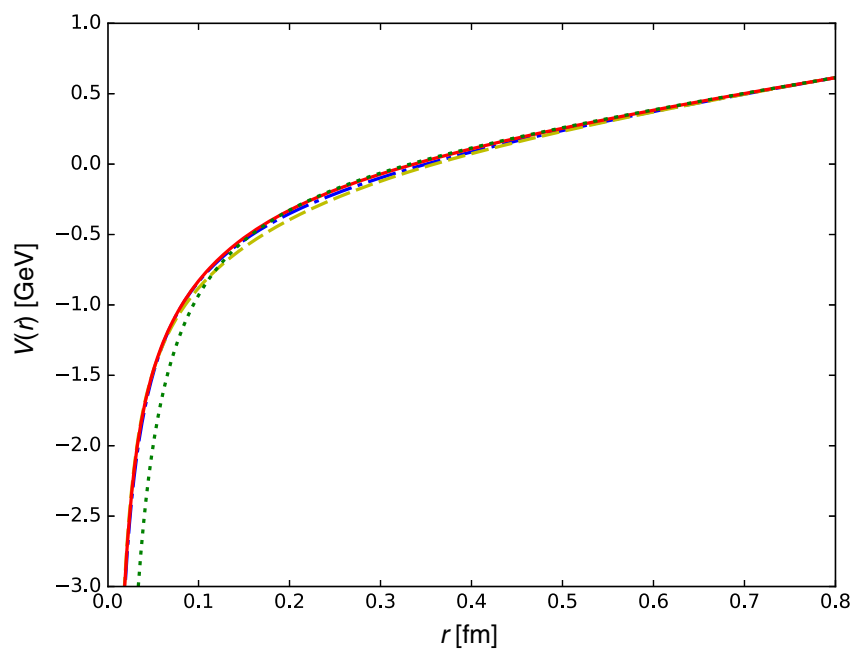

FIG. 2. Dependence of quarkonium potentials $V(r)$ on the interquark separation $r$. Our frozen- $\alpha_{\mathrm{s}}$ potential is shown in the solid red curve. The Cornell potential (green dots) [22], Richardson potential (blue dash-dotted) [27], and an alternative version of the new potential with $\mu=0.8 \mathrm{GeV}$ (gold dashes) are shown for comparison. 
TABLE I. $\quad P$-state masses [12] and splittings, in MeV.

\begin{tabular}{lrrr}
\hline \hline State & \multicolumn{1}{c}{$2 P(c \bar{c})$} & \multicolumn{1}{c}{$2 P(b \bar{b})$} & \multicolumn{1}{c}{$3 P(b \bar{b})$} \\
\hline$\chi_{0}\left({ }^{3} P_{0}\right)$ & $3414.71 \pm 0.30$ & $9859.44 \pm 0.52$ & $10232.5 \pm 0.64$ \\
$\chi_{1}\left({ }^{3} P_{1}\right)$ & $3510.67 \pm 0.05$ & $9892.78 \pm 0.4$ & $10255.46 \pm 0.55$ \\
$h\left({ }^{1} P_{1}\right)$ & $3525.38 \pm 0.11$ & $9899.3 \pm 0.8$ & $10259.8 \pm 1.12$ \\
$\chi_{2}\left({ }^{3} P_{2}\right)$ & $3556.17 \pm 0.07$ & $9912.21 \pm 0.4$ & $10268.85 \pm 0.55$ \\
${ }^{3} P_{J}$ centroid, $\langle\chi\rangle$ & $3525.29 \pm 0.01$ & $9899.87 \pm 0.17$ & $10260.35 \pm 0.31$ \\
$h-\langle\chi\rangle$ & $0.09 \pm 0.11$ & $-0.57 \pm 0.82$ & $-0.55 \pm 1.25$ \\
$\chi_{1}\left({ }^{3} P_{1}\right)-\chi_{0}\left({ }^{3} P_{0}\right)$ & $95.96 \pm 0.30$ & $33.34 \pm 0.66$ & $22.96 \pm 0.84$ \\
$\chi_{2}\left({ }^{3} P_{2}\right)-\chi_{0}\left({ }^{3} P_{2}\right)$ & $45.5 \pm 0.09$ & $19.43 \pm 0.57$ & $13.39 \pm 0.78$ \\
\hline \hline
\end{tabular}

Cornell potentials. All coincide at large distances. The Cornell potential is deeper at short distances than any of the potentials that take account of the evolution of $\alpha_{\mathrm{s}}$. For the convenience of others who may wish to apply the new potential, we present values of $\alpha_{\mathrm{s}}(r)$ suitable for interpolation in the Appendix.

We presented the general formalism for spin-dependent interactions as laid out by Eichten and Feinberg [5] and Gromes [32] in Sec. II B of Ref. [6], where we took a perturbative approach to the spin-orbit and tensor interactions. In the intervening time, the charmonium and bottomonium spectra have been mapped in detail, as summarized in Table I. This wealth of information leads us now to choose a more phenomenological approach.

We write the spin-dependent contributions to the $(c \bar{b})$ masses as

$$
\Delta=\sum_{k=1}^{4} T_{k},
$$

where the individual terms are

$$
\begin{aligned}
& T_{1}=\frac{\left\langle\vec{L} \cdot \vec{s}_{i}\right\rangle}{2 m_{i}^{2}} \tilde{T}_{1}\left(m_{i}, m_{j}\right)+\frac{\left\langle\vec{L} \cdot \vec{s}_{j}\right\rangle}{2 m_{j}^{2}} \tilde{T}_{1}\left(m_{j}, m_{i}\right) \\
& T_{2}=\frac{\left\langle\vec{L} \cdot \vec{s}_{i}\right\rangle}{m_{i} m_{j}} \tilde{T}_{2}\left(m_{i}, m_{j}\right)+\frac{\left\langle\vec{L} \cdot \vec{s}_{j}\right\rangle}{m_{i} m_{j}} \tilde{T}_{2}\left(m_{j}, m_{i}\right) \\
& T_{3}=\frac{\left\langle\vec{s}_{i} \cdot \vec{s}_{j}\right\rangle}{m_{i} m_{j}} \tilde{T}_{3}\left(m_{i}, m_{j}\right) \\
& T_{4}=\frac{\left\langle S_{i j}\right\rangle}{m_{i} m_{j}} \tilde{T}_{4}\left(m_{i}, m_{j}\right),
\end{aligned}
$$

$\vec{s}_{i}$ and $\vec{s}_{j}$ are the heavy-quark spins, $\vec{S}=\vec{s}_{i}+\vec{s}_{j}$ is the total spin, $\vec{L}$ is the orbital angular momentum of quark and antiquark in the bound state, $S_{i j}=4\left[3\left(\vec{s}_{i} \cdot \hat{n}\right)\left(\vec{s}_{j} \cdot \hat{n}\right)-\vec{s}_{i} \cdot \vec{s}_{j}\right]$ is the tensor operator, and $\hat{n}$ is an arbitrary unit vector.

We will deal with the hyperfine interaction $T_{3}$ momentarily. We express the other $\tilde{T}_{k}$ as

$$
\begin{aligned}
& \tilde{T}_{1}\left(m_{i}, m_{j}\right)=-\left\langle\frac{1}{r} \frac{d V}{d R}\right\rangle+2 \tilde{T}_{2}\left(m_{i}, m_{j}\right) \\
& \tilde{T}_{2}\left(m_{i}, m_{j}\right)=\frac{4 \tilde{c}_{2}}{3}\left\langle\frac{\alpha_{\mathrm{s}}(r)}{r^{3}}\right\rangle \\
& \tilde{T}_{4}\left(m_{i}, m_{j}\right)=\frac{\tilde{c}_{4}}{3}\left\langle\frac{\alpha_{\mathrm{s}}(r)}{r^{3}}\right\rangle
\end{aligned}
$$

where we have introduced the phenomenological coefficients $\tilde{c}_{2}$ and $\tilde{c}_{4}$, which take the value unity in the perturbative approach.

We extract values of $\tilde{T}_{2}$ and $\tilde{T}_{4}$ for the observed levels that appear in Table I. These are shown as the boldface entries in Table II. Then, we combine the definitions in Eq. (6) with our calculated values of $\left\langle\alpha_{\mathrm{s}} / r^{3}\right\rangle$ to determine $\tilde{c}_{2}$ and $\tilde{c}_{4}$ in the $(c \bar{c})$ and $(b \bar{b})$ families. The geometric mean of these values is our estimate for the coefficients in the $c \bar{b}$ system. We insert these back into Eq. (6) to estimate the values of $\tilde{T}_{2}$ and $\tilde{T}_{4}$ for the $B_{c}$ family. For completeness, we include our evaluations of $\langle(1 / r) d V / d r\rangle$ in the table.

For the $J / \psi$ and $\Upsilon$ families, composed of equal-mass heavy quarks, the familiar $L S$ coupling scheme, in which states are labeled by $n^{2 S+1} L_{J}$, is apt. When the quark

\begin{tabular}{|c|c|c|c|c|c|c|}
\hline ystem & $\tilde{T}_{2}$ & $\tilde{T}_{4}$ & $\begin{array}{c}\left\langle\frac{\alpha_{\mathrm{s}}(r)}{r^{3}}\right\rangle \\
\left(\mathrm{GeV}^{3}\right)\end{array}$ & $\tilde{c}_{2}$ & $\tilde{c}_{4}$ & $\begin{array}{c}\left\langle\frac{1}{r} \frac{d V}{d r}\right\rangle \\
\left(\mathrm{GeV}^{3}\right)\end{array}$ \\
\hline & 8 & 0308 & 0.05 & 1.25 & 1.77 & 0.141 \\
\hline $1 P$ & 8 & 0.0835 & 0.220 & 0.82 & 0.99 & 0.383 \\
\hline P & .181 & 0.05 & 0.1 & 0.82 & 0.99 & 0.278 \\
\hline $1 P(c \bar{b})$ & 0.119 & 0.0388 & 0.0885 & 1.012 & 1.316 & 0.198 \\
\hline
\end{tabular}
masses are unequal, as in the case at hand, spin-dependent terms in the Hamiltonian mix the spin-singlet and spintriplet $J=L$ states. We define

TABLE II. Values of $\tilde{T}_{2}$ and $\tilde{T}_{4}$ extracted from data (bold) and the inferred values of the phenomenological coefficients $\tilde{c}_{2}$ and $\tilde{c}_{4}$ for the $J / \psi$ and $\Upsilon$ families, from which the coefficients for the $(c \bar{b})$ system are derived. 


$$
\begin{aligned}
\left|n L_{L}\right\rangle^{\prime} & =\cos \theta\left|n^{1} L_{L}\right\rangle+\sin \theta\left|n^{3} L_{L}\right\rangle \\
\left|n L_{L}\right\rangle & =-\sin \theta\left|n^{1} L_{L}\right\rangle+\cos \theta\left|n^{3} L_{L}\right\rangle
\end{aligned}
$$

where

$$
\tan \theta=\frac{2 A}{B+\sqrt{B^{2}+4 A^{2}}}
$$

with

$$
A=\frac{1}{4} \sqrt{L(L+1)}\left(\frac{1}{m_{c}^{2}}-\frac{1}{m_{b}^{2}}\right) \tilde{T}_{1}
$$

and

$$
B=\frac{1}{4}\left(\frac{1}{m_{c}^{2}}+\frac{1}{m_{b}^{2}}\right) \tilde{T}_{1}+\frac{1}{m_{c} m_{b}} \tilde{T}_{2}-2 \frac{1}{m_{c} m_{b}} \tilde{T}_{4}
$$

Then our calculations of the $\tilde{T}_{k}$ defined in Eq. (6) lead to these values for the mixing angle: $\theta_{2 P}=18.7^{\circ}$, $\theta_{3 D}=-49.2^{\circ}, \theta_{3 P}=21.2^{\circ}, \theta_{4 F}=-49.5^{\circ}, \theta_{4 D}=-40.3^{\circ}$. A lattice calculation in quenched QCD [33] gave $\theta_{2 P}=33 \pm 2^{\circ}$.

The masses of the mixed states are

$$
\begin{aligned}
& M\left(n L_{L}^{\prime}\right)=\langle M(n L)\rangle-\frac{1}{2}\left(B-\sqrt{4 A^{2}+B^{2}}\right) \\
& M\left(n L_{L}\right)=\langle M(n L)\rangle-\frac{1}{2}\left(B+\sqrt{4 A^{2}+B^{2}}\right)
\end{aligned}
$$

where $\langle M(n L)\rangle$ is the $n L$ centroid.

At lowest order, the hyperfine splitting (HFS) between $s$ wave states, arising from $T_{3}$, is given by

$$
\Delta_{\mathrm{HFS}}^{(n)}=M\left(n^{3} S_{1}\right)-M\left(n^{1} S_{0}\right)=\frac{8 \alpha_{\mathrm{s}}\left|R_{n 0}(0)\right|^{2}}{9 m_{c} m_{b}},
$$

which is susceptible to significant quantum corrections. Rather than make a priori calculations of the hyperfine splitting, we adopt the lattice QCD result for the ground state and scale the splittings of excited states according to

$$
\frac{\Delta_{\mathrm{HFS}}^{(n)}}{\Delta_{\mathrm{HFS}}^{(1)}}=\frac{\left|R_{n 0}(0)\right|^{2}}{\left|R_{10}(0)\right|^{2}} .
$$

\section{THE $B_{c}$ SPECTRUM}

The vector meson $B_{c}^{*}$, the $1^{3} S_{1}$ hyperfine partner of $B_{c}$ and analog of $J / \psi$ and $\Upsilon$, has not yet been observed. Modern lattice calculations [14,34,35] give consistent values for the hyperfine splitting $M\left(B_{c}^{*}\right)-M\left(B_{c}\right)=$ $(53 \pm 7,54 \pm 7,55 \pm 3 \mathrm{MeV})$, so we take the mass of the vector state to be $M\left(B_{c}^{*}\right)=6329 \mathrm{MeV}$ and fix the centroid $\langle M(1 S)\rangle$ of the ground-state $s$-wave doublet at 6315.5 MeV for the lattice.
TABLE III. Calculated excitation energies (in $\mathrm{MeV}$ ) for $(c \bar{b})$ levels with respect to the $B_{c}(1 S)$ centroid according to potential models and lattice QCD simulations. The potential models have been aligned with the $1 S$ doublet centroid at $6315.5 \mathrm{MeV}$. Communication with states above flavor threshold is neglected.

\begin{tabular}{lccc}
\hline \hline Level & EQ94 [6] & Lattice QCD & This work \\
\hline $1^{1} S_{0}$ & -54.8 & $-40.5[14,34,35]$ & -40.5 \\
$1^{3} S_{1}$ & 18.2 & $13.5[14,34,35]$ & 13.5 \\
$2^{3} P_{0}$ & 381 & $393(17)(7)[35]$ & 377 \\
$2 P_{1}{ }^{\prime}$ & 411 & $417(18)(7)[35]$ & 415 \\
$2 P_{1}{ }^{\prime}$ & 417 & $446(30)[33]$ & 423 \\
$2^{3} P_{2}$ & 428 & $464(30)[33]$ & 435 \\
$2^{1} S_{0}$ & 537 & $561(18)(1)[14]$ & 551 \\
$2^{3} S_{1}$ & 580 & $601(19)(1)[14]$ & 582 \\
$3^{3} D_{1}$ & 693 & $\ldots$ & 691 \\
$3 D_{2}$ & 693 & $\ldots$ & 690 \\
$3 D_{2}^{\prime}$ & 686 & $\ldots$ & 700 \\
$3^{3} D_{3}$ & 690 & $\ldots$ & 695 \\
$3^{3} P_{0}$ & 789 & $\ldots$ & 789 \\
$3 P_{1}$ & 823 & $\ldots$ & 820 \\
$3 P^{\prime}$ & 816 & $\ldots$ & 828 \\
$3^{3} P_{2}$ & 834 & $\ldots$ & 839 \\
$3^{1} S_{0}$ & 925 & $\ldots$ & 938 \\
$3^{3} S_{1}$ & 961 & $\ldots$ & 964 \\
$4^{3} F_{2}$ & $\ldots$ & $\ldots$ & 918 \\
$4 F_{3}$ & $\ldots$ & $\ldots$ & 906 \\
$4 F_{3}^{\prime}$ & $\ldots$ & $\ldots$ & 922 \\
$4^{3} F_{4}$ & $\ldots$ & $\ldots$ & 908 \\
$4^{3} D_{1}$ & $\ldots$ & $\ldots$ & 1031 \\
$4 D_{2}$ & $\ldots$ & $\ldots$ & 1033 \\
$4 D_{2}^{\prime}$ & $\ldots$ & $\ldots$ & 1040 \\
$4^{3} D_{3}$ & $\ldots$ & $\ldots$ & 1038 \\
$4^{3} P_{0}$ & $\ldots$ & $\ldots$ & 1121 \\
$4 P_{1}$ & $\ldots$ & $\ldots$ & 1149 \\
$4 P_{1}^{\prime}$ & $\ldots$ & $\ldots$ & 1158 \\
$4^{3} P_{2}$ & $\ldots$ & $\ldots$ & 1257 \\
$4^{1} S_{0}$ & 1243 & $\ldots$ & 1280 \\
$4^{3} S_{1}$ & 1276 & $\ldots$ & \\
\hline \hline & & $\ldots$ & $\ldots$ \\
\hline
\end{tabular}

We summarize in Table III predictions for the spectrum of mesons with beauty and charm from our 1994 article [6], lattice QCD calculations, and the present work, expressed as excitations with respect to the $1 S$ centroid. Other potential-model calculations, some incorporating relativistic effects, may be found in the works cited in Ref. [7].

Our expectations for the spectrum of states are shown in the Grotrian diagram, Fig. 3, along with several of the lowest-lying open-flavor thresholds. The thresholds for strong decays of excited $(c \bar{b})$ levels are known experimentally to high accuracy, as shown in Table IV. Comparing with the model calculations summarized in Table III, we conclude that two sets of narrow $s$-wave $(c \bar{b})$ levels will lie below the beauty + charm flavor threshold, in agreement with general arguments [36]. All of the potential models cited in Ref. [7] predict $3^{3} S_{1}$ masses well above the 


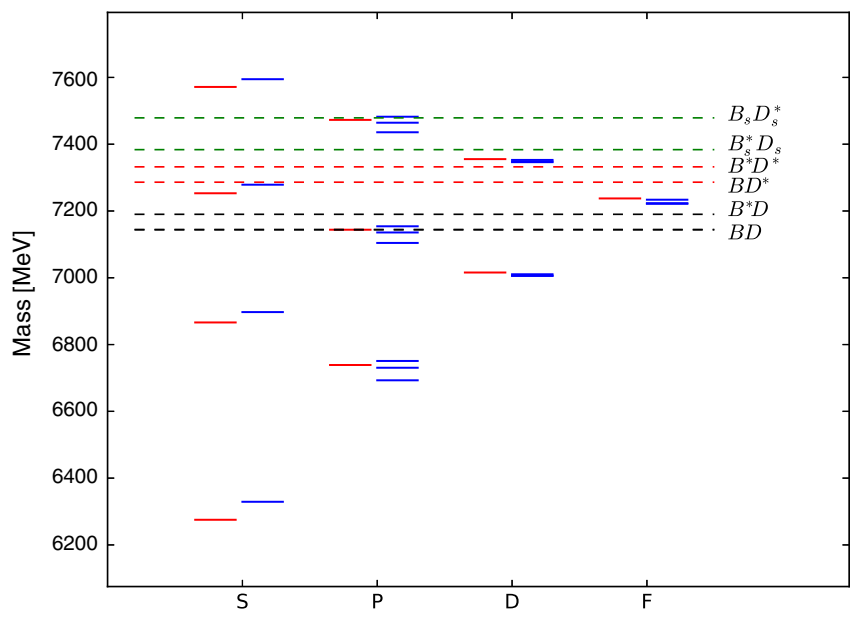

FIG. 3. Calculated $c \bar{b}$ spectrum, with (spin-singlet, spin-triplet) states shown on the left (right) in red (blue) for each orbitalangular-momentum family S, P, D, F. Dashed lines indicate thresholds for decay into two-body open-flavor channels given in Table IV.

$829-\mathrm{MeV} B D$ threshold. For the $3{ }^{1} S_{0}$ level, only the Ebert et al. prediction does not lie significantly above the $B^{*} D$ threshold. Lattice QCD calculations do not yet exist for states beyond the $2 S$ levels.

\section{DECAYS OF NARROW $(c \bar{b})$ LEVELS}

\section{A. Electromagnetic transitions}

The only significant decay mode for the $1^{3} S_{1}\left(B_{c}^{*}\right)$ state is the magnetic dipole (spin-flip) transition to the ground state, $B_{c}$. The M1 rate for transitions between $s$-wave levels is given by

$\Gamma_{\mathrm{M} 1}(i \rightarrow f+\gamma)=\frac{16 \alpha}{3} \mu^{2} k^{3}\left(2 J_{f}+1\right)\left|\left\langle f\left|j_{0}(k r / 2)\right| i\right\rangle\right|^{2}$,

TABLE IV. Open-flavor $(c \bar{b})$ thresholds and excitations above $1 S$ centroid, $6315.5 \mathrm{MeV}$, for $B_{c}$ levels, in $\mathrm{MeV}$.

\begin{tabular}{lcc}
\hline \hline State & Flavor threshold & Excitation energy \\
\hline$B^{+} D^{0}$ & $7144.15 \pm 0.15$ & 829 \\
$B^{0} D^{+}$ & $7149.28 \pm 0.16$ & 834 \\
$B^{*+} D^{0}$ & $7189.48 \pm 0.26$ & 874 \\
$B^{* 0} D^{+}$ & $7194.30 \pm 0.26$ & 879 \\
$B^{+} D^{* 0}$ & $7286.17 \pm 0.15$ & 971 \\
$B^{0} D^{*+}$ & $7289.89 \pm 0.16$ & 974 \\
$B^{*+} D^{* 0}$ & $7331.50 \pm 0.26$ & 1016 \\
$B^{* 0} D^{*+}$ & $7334.91 \pm 0.26$ & 1019 \\
$B_{s}^{0} D_{s}^{+}$ & $7335.23 \pm 0.20$ & 1020 \\
$B_{s}^{0} D_{s}^{*+}$ & $7479.09 \pm 0.44$ & 1164 \\
$B_{s}^{* 0} D_{s}^{+}$ & $7383.74_{-1.50}^{+1.80}$ & 1068 \\
$B_{s}^{* 0} D_{s}^{*+}$ & $7527.60_{-1.55}^{+1.84}$ & 1212 \\
\hline \hline
\end{tabular}

where the magnetic dipole moment is

$$
\mu=\frac{m_{b} e_{c}-m_{c} e_{\bar{b}}}{4 m_{c} m_{b}}
$$

and $k$ is the photon energy.

Apart from that M1 transition, only the electric dipole transitions are important for mapping the $(c \bar{b})$ spectrum. The strength of the electric-dipole transitions is governed by the size of the radiator and the charges of the constituent quarks. The E1 transition rate is given by

$\Gamma_{\mathrm{E} 1}(i \rightarrow f+\gamma)=\frac{4 \alpha\left\langle e_{Q}\right\rangle^{2}}{27} k^{3}\left(2 J_{f}+1\right)|\langle f|r| i\rangle|^{2} \mathcal{S}_{i f}$,

where the mean charge is

$$
\left\langle e_{Q}\right\rangle=\frac{m_{b} e_{c}-m_{c} e_{\bar{b}}}{m_{b}+m_{c}}
$$

$k$ is the photon energy, and the statistical factor $\mathcal{S}_{i f}=\mathcal{S}_{f i}$ is as defined by Eichten and Gottfried [37]. $\mathcal{S}_{\text {if }}=1$ for ${ }^{3} S_{1} \rightarrow{ }^{3} P_{J}$ transitions and $\mathcal{S}_{i f}=3$ for allowed E1 transitions between spin-singlet states. The statistical factors for $d$-wave to $p$-wave transitions are reproduced in Table $\mathrm{V}$.

The significant M1 and E1 electromagnetic transition rates and the $\pi \pi$ cascade rates are given in Table VI, along with the total widths in the absence of fission decays.

\section{B. Hadronic transitions}

We evaluate the rates for hadronic transitions between $(c \bar{b})$ levels according to the prescription we detailed in Sec. III B of Ref. [6]. The results are included in Table VI. Dipion cascades to the ground-state doublet are the dominant decay modes of $2^{3} S_{1}$ and $2^{1} S_{0}$ and will be key to characterizing those states, as we shall discuss in Sec. VA.

As observed long ago by Brown and Cahn [38], an amplitude zero imposed by chiral symmetry pushes the $\pi^{+} \pi^{-}$invariant mass distribution to higher invariant masses than phase space alone would predict. In its simplest form, this analysis yields a universal form for the normalized dipion invariant mass distribution in quarkonium cascades $\Phi^{\prime} \rightarrow \Phi \pi^{+} \pi^{-}$,

TABLE V. Statistical factor $\mathcal{S}_{i f}=\mathcal{S}_{f i}$ for ${ }^{3} P_{J} \rightarrow{ }^{3} D_{J^{\prime}}+\gamma$ and ${ }^{3} D_{J} \rightarrow{ }^{3} P_{J^{\prime}}+\gamma$ transitions.

\begin{tabular}{lcc}
\hline \hline$J$ & $J^{\prime}$ & $\mathcal{S}_{i f}$ \\
\hline 0 & 1 & 2 \\
1 & 1 & $1 / 2$ \\
1 & 2 & $9 / 10$ \\
2 & 1 & $1 / 50$ \\
2 & 2 & $9 / 50$ \\
2 & 3 & $18 / 25$ \\
\hline \hline
\end{tabular}


TABLE VI. Total widths $\Gamma$ and branching fractions $\mathcal{B}$ for principal decay modes of $(c \bar{b})$ states below threshold, updating Table IX of Ref. [6]. Dissociation into $B D$, etc., will dominate over the tabulated decay modes for states above threshold.

\begin{tabular}{|c|c|c|}
\hline Decay mode & $k_{\gamma}(\mathrm{keV})$ & $\begin{array}{c}\text { Branching } \\
\text { fraction }(\%)\end{array}$ \\
\hline
\end{tabular}

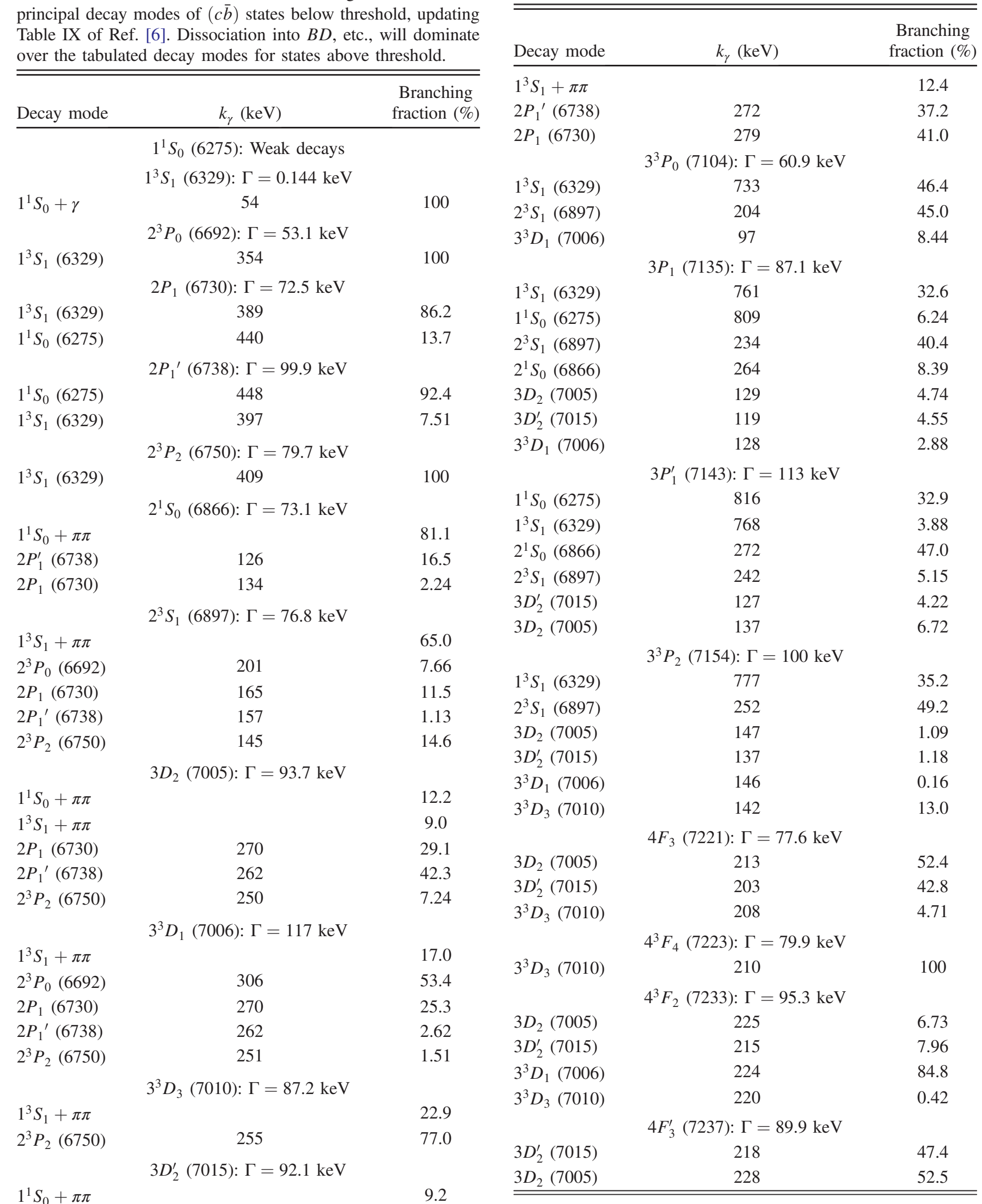

TABLE VI. (Continued) 


$$
\frac{1}{\Gamma} \frac{d \Gamma}{d \mathcal{M}}=\text { constant } \times \frac{|\vec{K}|}{M_{\Phi^{\prime}}^{2}}\left(2 x^{2}-1\right)^{2} \sqrt{x^{2}-1},
$$

where $x=\mathcal{M} / 2 m_{\pi}$ and $\vec{K}$ is the three-momentum carried by the pion pair. The soft-pion expression (18) describes the depletion of the dipion spectrum at low invariant masses observed in the transitions $\psi(2 S) \rightarrow \psi(1 S) \pi \pi, \Upsilon(2 S) \rightarrow$ $\Upsilon(1 S) \pi \pi$, and $\Upsilon(3 S) \rightarrow \Upsilon(2 S) \pi \pi$, but fails to account for structures in the $\Upsilon(3 S) \rightarrow \Upsilon(1 S) \pi \pi$ spectrum [39]. We expect the $3 S$ levels to lie above flavor threshold in the $(c \bar{b})$ system and so to have very small branching fractions for cascade decays (but see the final paragraph of Sec. VA).

\section{Properties of $(c \bar{b})$ wave functions at the origin}

For quarks bound in a central potential, it is convenient to separate the Schrödinger wave function into radial and angular pieces, as $\Psi_{n \ell m}(\vec{r})=R_{n \ell}(r) Y_{\ell m}(\theta, \phi)$, where $n$ is the principal quantum number, $\ell$ and $m$ are the orbital angular momentum and its projection, $R_{n \ell}(r)$ is the radial wave function, and $Y_{\ell m}(\theta, \phi)$ is a spherical harmonic [40]. The Schrödinger wave function is normalized, $\int d^{3} \vec{r}\left|\Psi_{n \ell m}(\vec{r})\right|^{2}=1$, so that $\int_{0}^{\infty} r^{2} d r\left|R_{n \ell}(r)\right|=1$. The value of the radial wave function, or its first nonvanishing derivative, at the origin,

$$
\left.R_{n \ell}^{(\ell)}(0) \equiv \frac{d^{\ell} R_{n \ell}(r)}{d r^{\ell}}\right|_{r=0},
$$

is required to evaluate pseudoscalar decay constants and production rates through heavy-quark fragmentation. Our calculated values of $\left|R_{n \ell}^{(\ell)}(0)\right|^{2}$ are given in Table VII.

The pseudoscalar decay constant $f_{B_{c}}$, which enters the calculations of annihilation decays such as $c \bar{b} \rightarrow W^{+} \rightarrow \tau^{+}+$ $\nu_{\tau}$, is defined by

$$
\left\langle 0\left|A_{\mu}(0)\right| B_{c}(q)\right\rangle=i f_{B_{c}} \mathrm{~V}_{c b} q_{\mu},
$$

where $A_{\mu}$ is the axial-vector part of the charged weak current, $\mathrm{V}_{c b}$ is an element of the Cabibbo-Kobayashi-Maskawa quark-mixing matrix, and $q_{\mu}$ is the four-momentum of the $B_{c}$. Its counterpart for the vector state is

$$
\left\langle 0\left|V_{\mu}(0)\right| B_{c}^{*}(q)\right\rangle=i f_{B_{c}^{*}} \mathrm{~V}_{c b} \varepsilon_{\mu}^{*},
$$

where $V_{\mu}$ is the vector part of the charged weak current and $\varepsilon_{\mu}^{*}$ is the polarization vector of the $B_{c}^{*}$. The ground-state pseudoscalar and vector decay constants are given in terms of the wave function at the origin by the Van RoyenWeisskopf formula [41], generically

$$
f_{B_{c}^{(*)}}^{2}=\frac{3\left|R_{10}(0)\right|^{2}}{\pi M} \bar{C}^{2}\left(\alpha_{\mathrm{s}}\right),
$$

where the leading-order QCD correction is given by [42]
TABLE VII. Squares of radial wave functions at the origin and related quantities [cf. Eq. (19)] for $(c \bar{b})$ mesons.

\begin{tabular}{ll}
\hline \hline Level & \multicolumn{1}{c}{$\left|R_{n \ell}^{(\ell)}(0)\right|^{2}$} \\
\hline $1 S$ & $1.994 \mathrm{GeV}^{3}$ \\
$2 P$ & $0.3083 \mathrm{GeV}^{5}$ \\
$2 S$ & $1.144 \mathrm{GeV}^{3}$ \\
$3 D$ & $0.0986 \mathrm{GeV}^{7}$ \\
$3 P$ & $0.3939 \mathrm{GeV}^{5}$ \\
$3 S$ & $0.9440 \mathrm{GeV}^{3}$ \\
$4 F$ & $0.0493 \mathrm{GeV}^{9}$ \\
$4 D$ & $0.1989 \mathrm{GeV}^{7}$ \\
$4 P$ & $0.4540 \mathrm{GeV}^{5}$ \\
$4 S$ & $0.8504 \mathrm{GeV}^{3}$ \\
\hline \hline
\end{tabular}

$$
\bar{C}^{2}\left(\alpha_{\mathrm{s}}\right)=1-\frac{\alpha_{\mathrm{s}}}{\pi}\left(\delta^{\mathrm{P}, \mathrm{V}}-\frac{m_{c}-m_{b}}{m_{c}+m_{b}} \ln \frac{m_{c}}{m_{b}}\right)
$$

and

$$
\delta^{\mathrm{P}}=2 ; \quad \delta^{\mathrm{V}}=8 / 3 .
$$

Choosing the representative value $\alpha_{\mathrm{s}}=0.38$, and using the quark masses given in Eq. (2), we find

$$
\bar{C}\left(\alpha_{\mathrm{s}}\right)=\begin{aligned}
& 0.904, \mathrm{P} \\
& 0.858, \mathrm{~V}
\end{aligned}
$$

Consequently, we estimate the ground-state meson decay constants as

$$
f_{B_{c}}=498 \mathrm{MeV} ; \quad f_{B_{c}^{*}}=471 \mathrm{MeV},
$$

so that $f_{B_{c}^{*}} / f_{B_{c}}=0.945$. The compact size of the $(c \bar{b})$ system enhances the pseudoscalar decay constant relative to $f_{\pi}$ and $f_{K}$.

This is to be compared to a state-of-the-art lattice evaluation [43], $f_{B_{c}}=434 \pm 15 \mathrm{MeV}$, which entails improved nonrelativistic QCD for the valence $b$-quark and the highly improved staggered quark action for the lighter quarks on gluon field configurations that include the effect of $u / d, s$, and $c$ quarks in the sea with the $u / d$ quark masses going down to physical values. The same calculation yields $f_{B_{c}^{*}} / f_{B_{c}}=0.988 \pm 0.027$. A calculation in the framework of QCD sum rules gives $f_{B_{c}}=528 \pm$ $19 \mathrm{MeV}$ [44].

\section{PRODUCTION OF $(c \bar{b})$ STATES AT THE LARGE HADRON COLLIDER}

We present in Table VIII cross sections for the production of $B_{c}$ states at the Large Hadron Collider, calculated using the framework of the BCVEGPY2.2 generator [45], which we have extended to include the production of $3 P$ 
TABLE VIII. Production rates (in nanobarn) for $(c \bar{b})$ states in $p p$ collisions at the LHC. The production rates were calculated using the BCVEGPY2.2 generator of Ref. [45], extended to include the production of $3 P$ states. Color-octet contributions to $s$-wave production are small; we show them (following |) only for the $1 S$ states.

\begin{tabular}{lccc}
\hline \hline $\begin{array}{c}(c \bar{b}) \\
\text { level }\end{array}$ & $\sigma(\sqrt{s}=8 \mathrm{TeV})$ & $\sigma(\sqrt{s}=13 \mathrm{TeV})$ & $\sigma(\sqrt{s}=14 \mathrm{TeV})$ \\
\hline $1^{1} S_{0}$ & $46.8 \mid 1.01$ & $80.3 \mid 1.75$ & $88.0 \mid 1.90$ \\
$1^{3} S_{1}$ & $123.0 \mid 4.08$ & $219.1 \mid 6.97$ & $237.0 \mid 7.55$ \\
$2^{3} P_{0}$ & 1.113 & 1.959 & 2.108 \\
$2^{3} P_{1}$ & 2.676 & 4.783 & 5.214 \\
$2^{1} P_{1}$ & 3.185 & 5.702 & 6.166 \\
$2^{3} P_{2}$ & 6.570 & 11.57 & 12.64 \\
$2^{1} S_{0}$ & 9.58 & 16.94 & 18.45 \\
$2^{3} S_{1}$ & 23.46 & 41.72 & 45.53 \\
$3^{3} P_{0}$ & 0.915 & 1.642 & 1.806 \\
$3^{3} P_{1}$ & 2.263 & 4.082 & 4.478 \\
$3^{1} P_{1}$ & 2.695 & 4.817 & 5.287 \\
$3^{3} P_{2}$ & 5.53 & 9.98 & 10.90 \\
$3^{1} S_{0}$ & 4.23 & 7.53 & 8.08 \\
$3^{3} S_{1}$ & 10.16 & 18.21 & 19.83 \\
\hline \hline
\end{tabular}

states. Cross sections for the physical $(2,3) P_{1}^{(\prime)}$ states are appropriately weighted mixtures of the ${ }^{3} P_{1}$ and ${ }^{1} P_{1}$ cross sections.

The rapidity distributions (for $B_{c}^{*}$ production, Fig. 4) and transverse-momentum distributions (shown for $B_{c}$ production, Fig. 5) are similar in character for $\sqrt{s}=8,13$, and $14 \mathrm{TeV}$. The rapidity distributions for low-lying $(c \bar{b})$ states

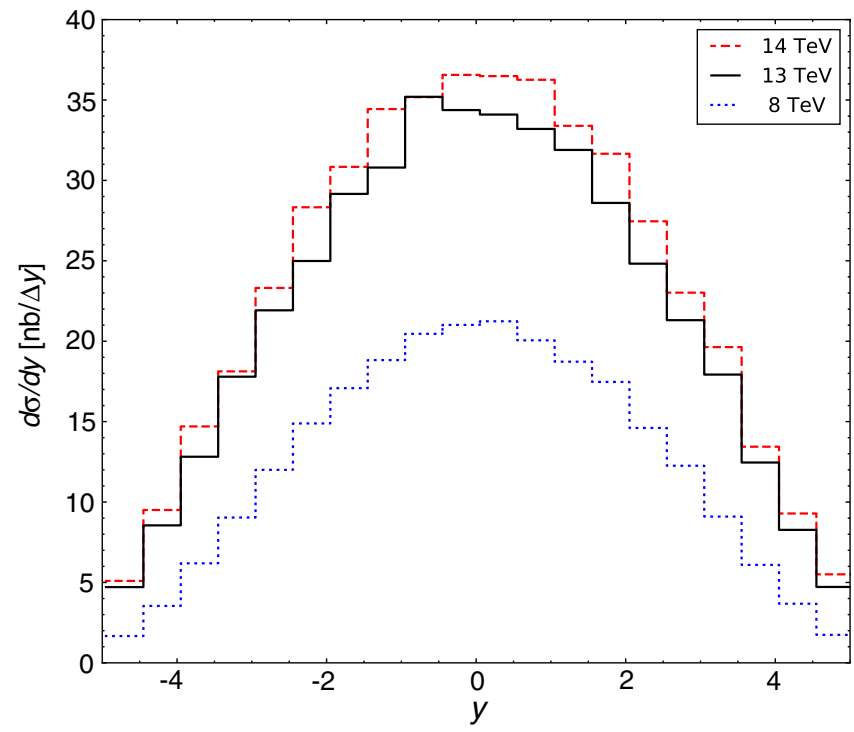

FIG. 4. Rapidity distribution for production of $B_{c}^{*}$ in $p p$ collisions at $\sqrt{s}=8 \mathrm{TeV}$ (dotted blue curve), $\sqrt{s}=13 \mathrm{TeV}$ (solid black curve), and $\sqrt{s}=14 \mathrm{TeV}$ (dashed red curve), calculated using BCVEGPY2.2 [45]. The bin width is $\Delta y=0.5$. The mild asymmetries are statistical fluctuations.

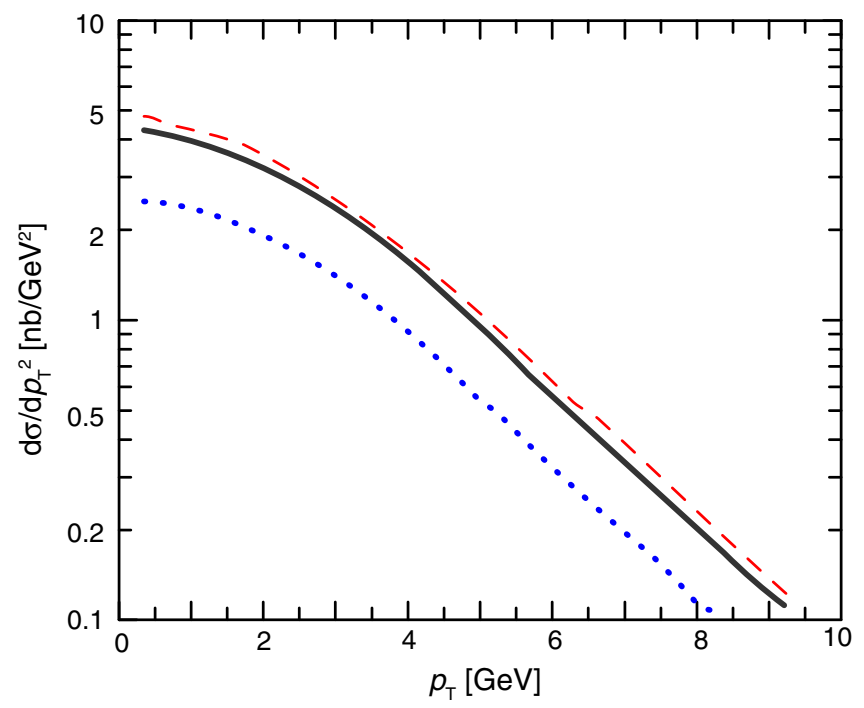

FIG. 5. Transverse momentum distribution of $B_{c}$ produced in $p p$ collisions at $\sqrt{s}=8 \mathrm{TeV}$ (dotted blue curve), $\sqrt{s}=13 \mathrm{TeV}$ (solid black curve), and $\sqrt{s}=14 \mathrm{TeV}$ (dashed red curve), calculated using BCVEGPY2.2 [45]. Small shape variations are statistical fluctuations.

are shown in Fig. 6. The acceptance of the CMS and ATLAS detectors covers central pseudorapidity $|\eta| \leq 2.5$, whereas the geometrical acceptance of the LHC $b$ detector is characterized by $2 \leq \eta \leq 5$. For comparison, approximately $68 \%$ of the $B_{c}^{*}$ cross section lies within $|y| \leq 2.5$, and approximately $22 \%$ is produced at forward rapidities $y>2$. Similar fractions hold for all the $(c \bar{b})$ levels.

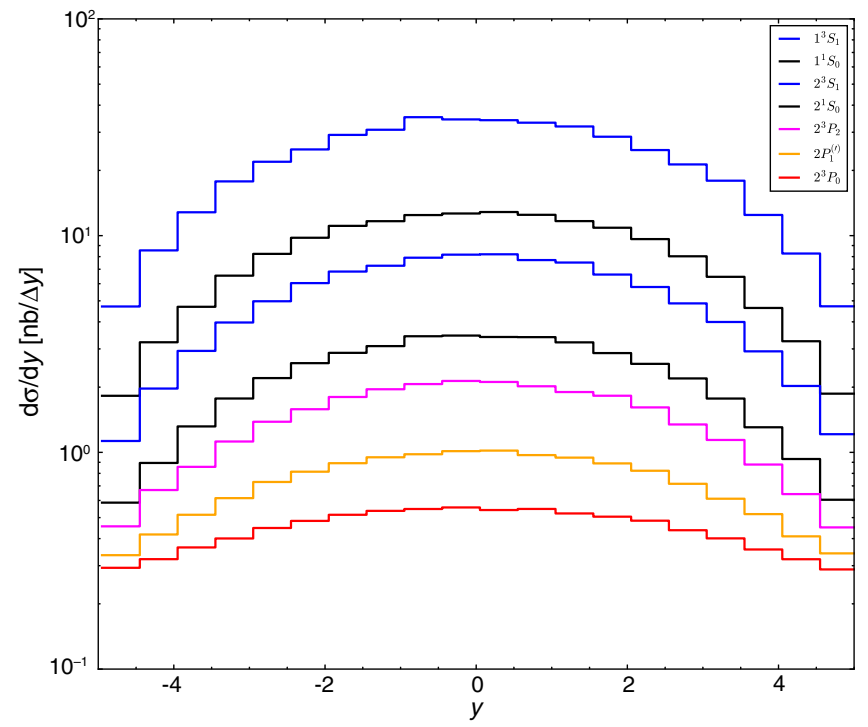

FIG. 6. Rapidity distributions for the production of low-lying $(c \bar{b})$ states in $p p$ collisions at $\sqrt{s}=13 \mathrm{TeV}$, calculated using BCVEGPY2.2 [45]. From highest to lowest, the histograms refer to production of the $1^{3} S_{1}, 1^{1} S_{0}, 2^{3} S_{1}, 2^{1} S_{0}, 2^{3} P_{2}, 2 P_{1^{(1)}}, 2^{3} P_{0}$ levels. 


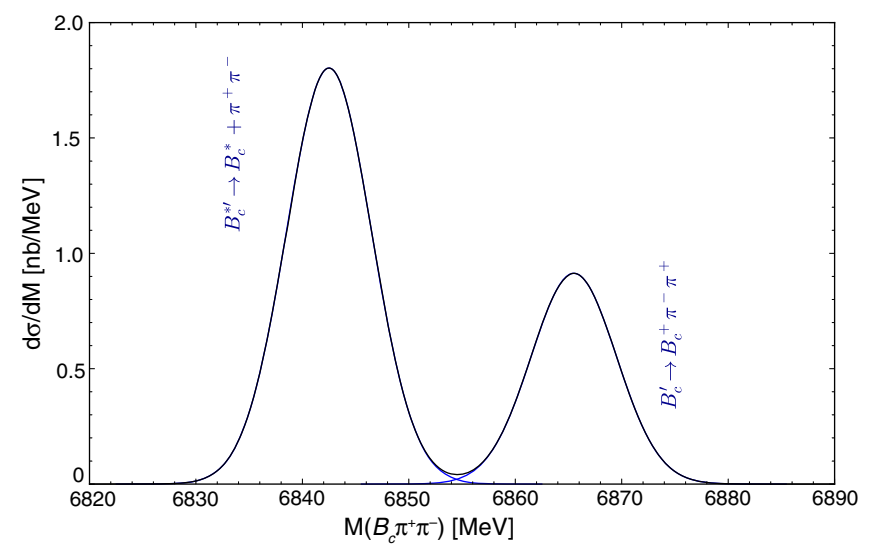

FIG. 7. Calculated positions and relative strengths of the twopion cascades $B_{c}(2 S) \rightarrow B_{c}(1 S) \pi^{+} \pi^{-}$, represented as Gaussian line shapes with standard deviation of $4 \mathrm{MeV}$. Production rates are given in Table VIII and branching fractions in Table VI. We assume that the photon in the transition $B_{c}^{*} \rightarrow B_{c}+\gamma$ is not included in the reconstruction. Rates confined to rapidity $|y| \leq$ 2.5 are $0.68 \times$ those shown.

\section{A. Dipion cascades}

The path to establishing excited states will proceed by resolving two separate peaks in the invariant mass distributions associated with the cascades $B_{c}^{\prime} \rightarrow B_{c} \pi^{+} \pi^{-}$and $B_{c}^{* \prime} \rightarrow B_{c}^{*}+\pi^{+} \pi^{-}, \quad B_{c}^{*} \rightarrow B_{c}+\gamma \quad$ (gamma unobserved). The splitting between the peaks is set by the difference of mass differences,

$$
\Delta_{21} \equiv\left[M\left(B_{c}^{* \prime}\right)-M\left(B_{c}^{\prime}\right)\right]-\left[M\left(B_{c}^{*}\right)-M\left(B_{c}\right)\right],
$$

generically expected to be negative [46]. The corresponding quantity is approximately $-64 \mathrm{MeV}$ in the $(c \bar{c})$ family and $-37 \mathrm{MeV}$ in the $(b \bar{b})$ family [12]. For the $(c \bar{b})$ system, a modern lattice simulation [14] gives $\Delta_{21}=-15 \mathrm{MeV}$, whereas the result of our potential-model calculation is $-23 \mathrm{MeV}$. In these circumstances, the undetected fourmomentum of the photon means that the reconstructed " $B_{c}^{*}$ " mass should correspond to the lower peak.

We show an example of what is to be expected in Fig. 7, taking the direct production cross sections (with no rapidity cuts) from Table VIII and the branching fractions from Table VI. The relative heights of (and relative number of events in) the peaks measures the ratio

$$
\mathcal{R} \equiv \frac{\sigma\left(B_{c}^{* \prime}+X\right) \mathcal{B}\left(B_{c}^{* \prime} \rightarrow B_{c}^{*}+\pi^{+} \pi^{-}\right)}{\sigma\left(B_{c}^{\prime}+X\right) \mathcal{B}\left(B_{c}^{\prime} \rightarrow B_{c}+\pi^{+} \pi^{-}\right)} .
$$

At $\sqrt{s}=13 \mathrm{TeV}$, the ratio of cross sections is nearly 2.5 . Taking account of the branching fractions, we estimate $\mathcal{R} \approx 2$. If $B_{c}^{*}$ and $B_{c}$ were produced with equal frequency, we would find $\mathcal{R} \approx 0.8$.

Now the CMS Collaboration [4] at the Large Hadron Collider, analyzing $140 \mathrm{fb}^{-1}$ of $p p$ collisions at $\sqrt{s}=13 \mathrm{TeV}$, has observed a pattern that closely resembles the template of Fig. 7. In the distribution of $M\left(B_{c} \pi^{+} \pi^{-}\right)-M\left(B_{c}\right)^{\text {obs }}+$ $M\left(B_{c}\right)$, they reconstruct a peak at $6871.0 \pm 1.2 \mathrm{MeV}$, which they identify as $B_{c}(2 S)$, and a second peak $29.0 \pm$ $1.5 \mathrm{MeV}$ lower in mass (statistical errors only). (The observed $B_{c}$ mass is replaced, event by event, with the world-average value to sharpen resolution.) The putative $B_{c}(2 S)$ lies within $5 \mathrm{MeV}$ of our expectation for the $2^{1} S_{0}$ level, and the separation is to be compared with our expectation of $23 \mathrm{MeV}$. If we impose the scaling relation Eq. (13) for the hyperfine splittings, we reproduce the observed 29-MeV separation with $M\left(B_{c}^{*}\right)-M\left(B_{c}\right)=$ $68 \mathrm{MeV}, M\left(B_{c}^{* \prime}\right)-M\left(B_{c}^{\prime}\right)=39 \mathrm{MeV}$. The $B_{c}^{*} \rightarrow B_{c}+\gamma$ photon momentum would be $68 \mathrm{MeV}$.

An unbinned extended maximum-likelihood fit to the CMS data returns $66 \pm 10$ events for the lower peak and $51 \pm 10$ for the upper. These yields are not yet corrected for detection efficiencies and acceptances, so they cannot be used to infer ratios of production cross sections times branching fractions. We look forward to the final result and to studies of the $\pi^{+} \pi^{-}$invariant mass distribution as next steps in $B_{c}$ spectroscopy.

Our calculations indicate that the $3 S$ levels will lie above flavor threshold (see Sec. V C, especially the discussion surrounding Figs. 9 and 10), but it is conceivable that coupledchannel effects might push one or both states lower in mass. For that reason, it is worth examining the $B_{c} \pi^{+} \pi^{-}$mass spectrum up through $7200 \mathrm{MeV}$ for indications of $3^{1} S_{0} \rightarrow$ $B_{c} \pi^{+} \pi^{-}$and $3^{3} S_{1} \rightarrow B_{c}^{*} \pi^{+} \pi^{-}$lines. According to our estimate of the $3 S$ hyperfine splitting, the $3^{3} S_{1}$ line would lie about $28 \mathrm{MeV}$ below the $3^{1} S_{0}$ line $(36 \mathrm{MeV}$ if we reset the $1 S$ splitting to $68 \mathrm{MeV})$. For orientation, note that $\mathcal{B}(\Upsilon(3 S) \rightarrow$ $\left.\Upsilon(1 S) \pi^{+} \pi^{-}\right)=4.37 \pm 0.08 \%$, while $36 \%$ of $\Upsilon(3 S)$ decays proceed through the $g g g$ channel, which is not available to the $(c \bar{b})$ states. According to Table VIII, the $3 S$ states are produced at approximately $44 \%$ of the rate for their $2 S$ counterparts.

\section{B. Electromagnetic transitions}

It may in time become possible for experiments to detect some of the more energetic E1-transition photons that appear in Table VI. As an incentive for the search, we show in Fig. 8 the spectrum of E1 photons in decays of the $2^{3} S_{1}$ and $2^{1} S_{0}$ levels as well as the $2 P \rightarrow 2 S$ transitions, assuming as always a missing $B_{c}^{*} \rightarrow B_{c} \gamma$ photon in the reconstruction. Here we include direct production of the $2 P$ states as well as feed-down from $2 S \rightarrow 2 P$ transitions. The strong $B_{c}^{*} \rightarrow B_{c}$ line arising from direct production of $B_{c}^{*}$, for which we calculate $\sigma \cdot \mathcal{B} \approx 225 \mathrm{nb}$ at $\sqrt{s}=13 \mathrm{TeV}$, is probably too low in energy to be observed. More promising are the $2 P$ levels, which might show themselves in $B_{c}+\gamma$ invariant mass distributions. These lines make up the righthand group (black lines) in Fig. 8. The $2^{3} P_{2}(6750) \rightarrow B_{c}^{*} \gamma$ line is a particularly attractive target for experiment because of the favorable production cross section, branching fraction, and $409-\mathrm{MeV}$ photon energy. The $2 P$ masses inferred from transitions to $B_{c}^{*}$ will be shifted downward because of the unobserved M1 photon. It is not possible to produce 


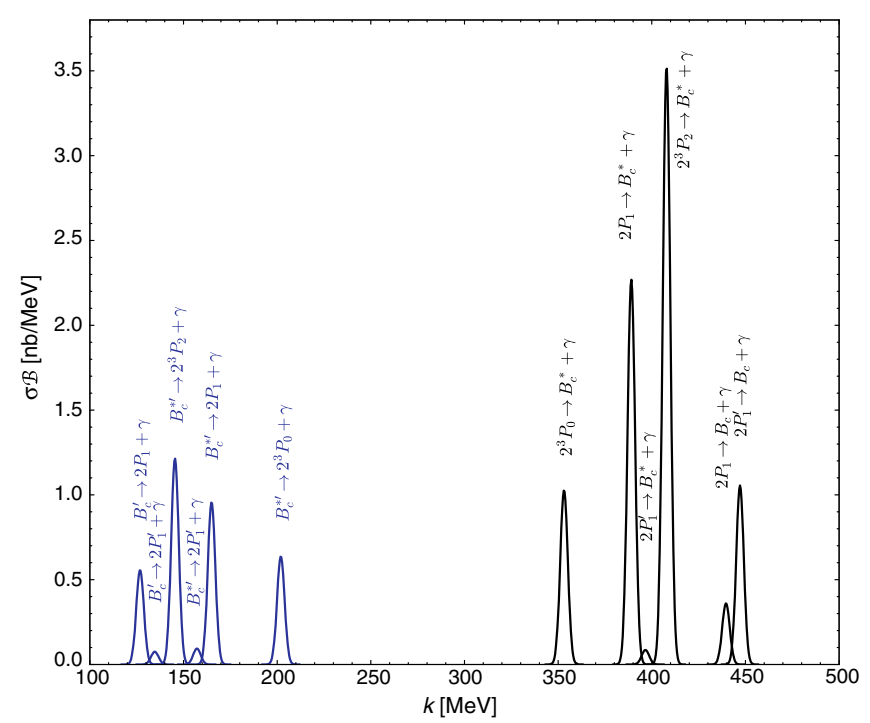

FIG. 8. Photon energies $k$ and relative strengths of E1 transitions from $2 S \rightarrow 2 P$ (left group, blue curves) and $2 P \rightarrow 1 S$ (right group, black curves) $(c \bar{b})$ states. Production rates are taken from Table VIII and branching fractions from Table VI. We suppose that the photon transition $B_{c}^{*} \rightarrow B_{c}+\gamma$ goes unobserved in the cascade transitions. We assume Gaussian line shapes with standard deviation $2 \mathrm{MeV}$.

enriched samples of the $2 S$ levels by tuning the energy of $e^{+} e^{-}$collisions, as is done for $J / \psi$ and $\Upsilon$, so reconstruction of the left-hand group of $2 S \rightarrow 2 P$ transitions (blue lines in Fig. 8) will be problematic.

In the far future, combining the photon transition energies and relative rates with expectations for production and decay may eventually make it possible to disentangle mixing of the spin-singlet and spin-triplet $J=L$ states.

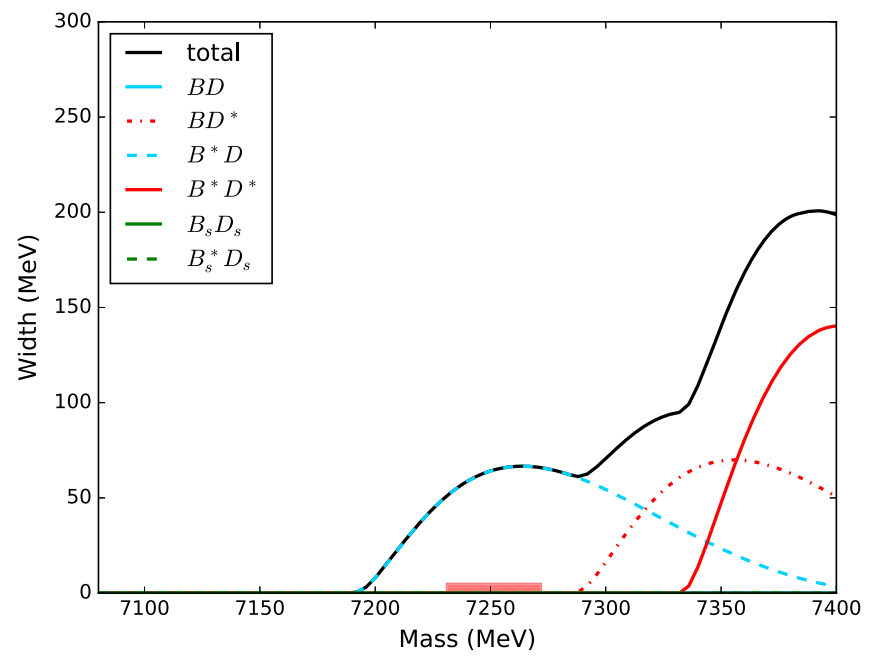

FIG. 9. Strong decay widths of the $3{ }^{1} S_{0}(c \bar{b})$ level near openflavor threshold. The shaded band on the mass axis indicates $\pm 20 \mathrm{MeV}$ around our nominal value for the mass of this state, $7253 \mathrm{MeV}$.

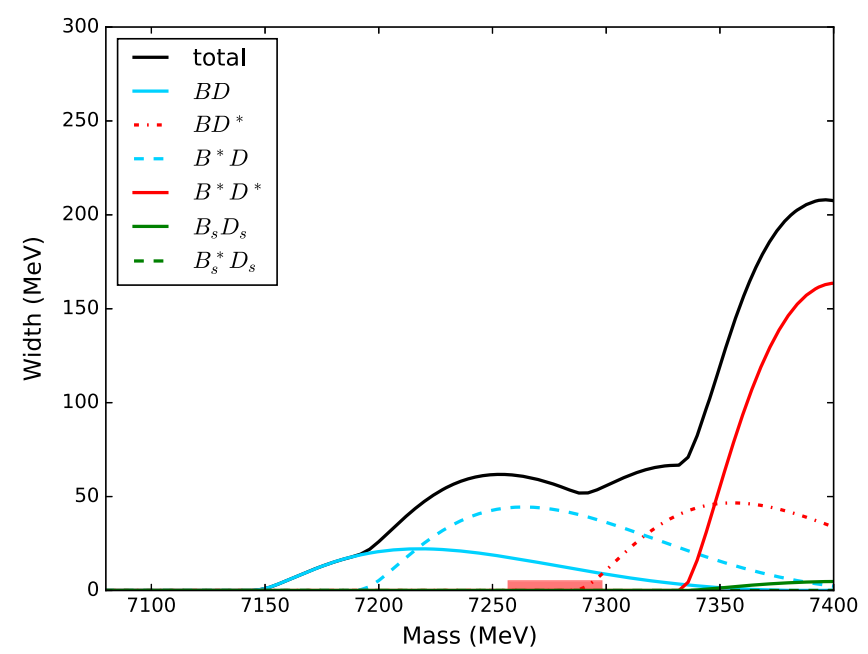

FIG. 10. Strong decay widths of the $3^{3} S_{1}(c \bar{b})$ level near openflavor threshold. The shaded band on the mass axis indicates $\pm 20 \mathrm{MeV}$ around our nominal value for the mass of this state, $7279 \mathrm{MeV}$.

\section{States above open-flavor threshold}

We estimate the strong decay rates for $(c \bar{b})$ states that lie above flavor threshold using the Cornell coupled-channel formalism [22] that we elaborated and applied to charmonium states in [23].

We expect both the $3^{1} S_{0}$ and $3^{3} S_{1}$ states to lie above threshold for strong decays. The $3^{1} S_{0}$ state can decay into the final state $B^{*} D$ and the $3^{3} S_{1}$ level can decay into both the $B D$ and $B^{*} D$ final states. The open decay channels as a function of the masses of these states is shown in Figs. 9 and 10.

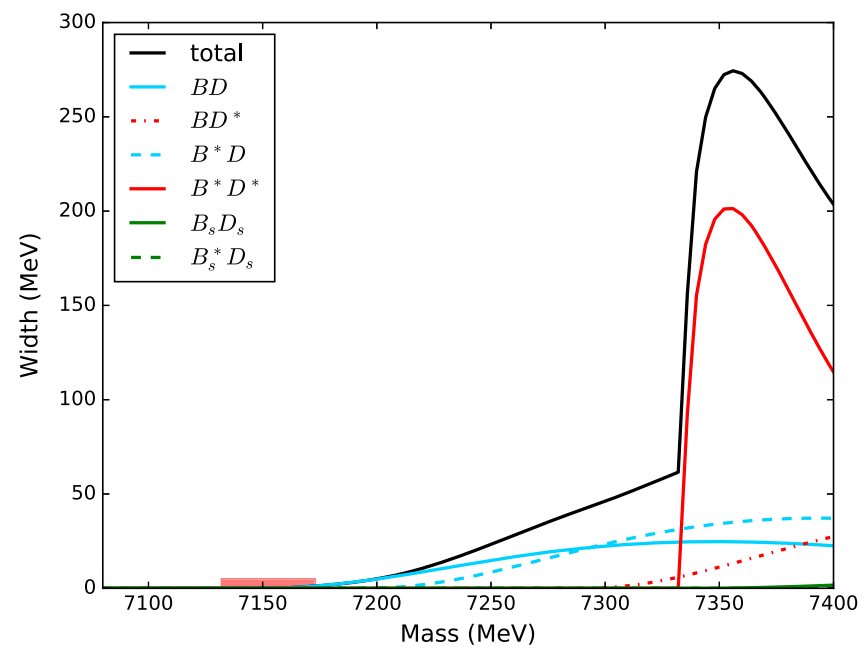

FIG. 11. Strong decay widths of the $3^{3} P_{2}(c \bar{b})$ level near openflavor threshold. The shaded band on the mass axis indicates $\pm 20 \mathrm{MeV}$ around our nominal value for the mass of this state, $7154 \mathrm{MeV}$. 


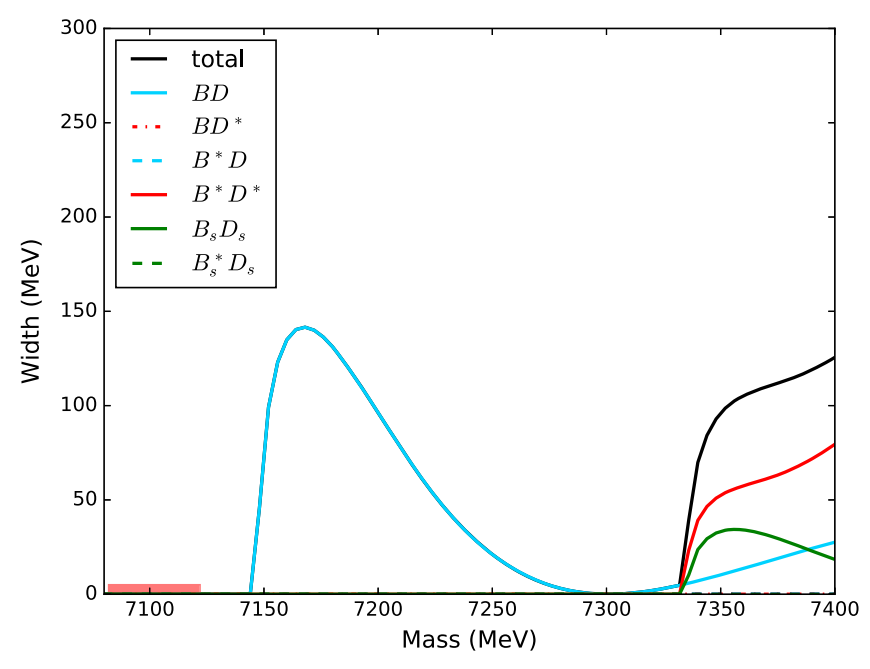

FIG. 12. Estimated strong decay widths of the $3^{3} P_{0}(c \bar{b})$ level near open-flavor threshold. The shaded band on the mass axis indicates $\pm 20 \mathrm{MeV}$ around our nominal value for the mass of this state, $7104 \mathrm{MeV}$.

The $3^{3} P_{2}$ state might be observed as a very narrow ( $d$-wave) $B D$ line near open-flavor threshold. Its decay width as a function of mass for the $2 P$ states is given in Fig. 11.

In the phenomenological models the remaining $3 P$ states lie just below the thresholds for strong decays. However, they are near enough to these thresholds that there might be interesting behavior at the threshold for $B^{*} D$ in the $3 P_{1}^{(\prime)}$ cases and for the $B D$ threshold in the case of the $3^{3} P_{0}$ state. Figure 12 shows that the $3^{3} P_{0}$ width grows rapidly just above threshold. The strong decay widths as a function of mass for the $3 P_{1}$ and $3 P_{1}^{\prime}$ states have a common behavior, displayed in Fig. 13.

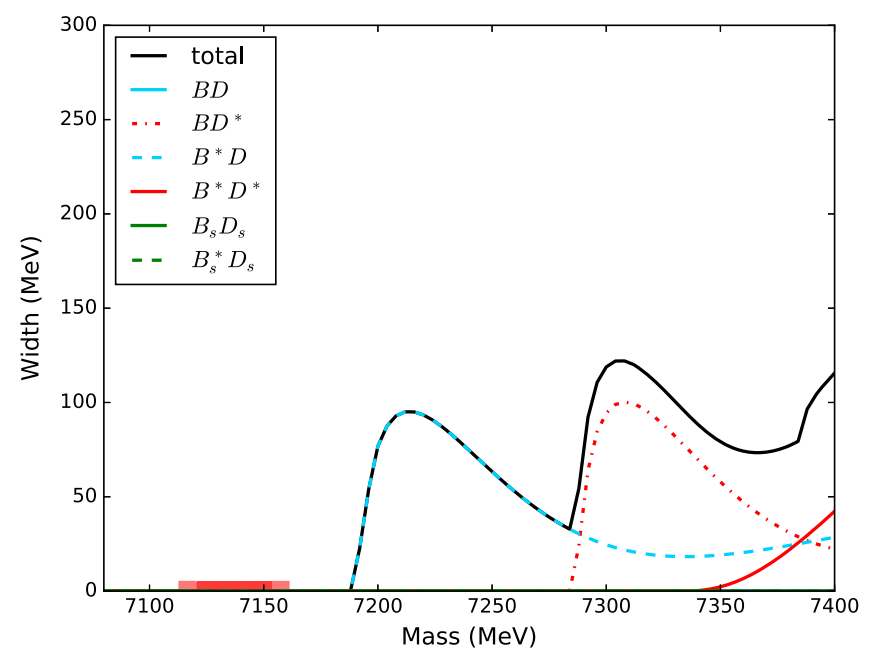

FIG. 13. Strong decay widths of the $3 P_{1}$ or $3 P_{1}^{(\prime)}$. The shaded band on the mass axis indicates $\pm 20 \mathrm{MeV}$ around our nominal values for the masses of these state, 7135 and $7143 \mathrm{MeV}$.
It is worth keeping in mind that while narrow $B D$ peaks may signal excited $(c \bar{b})$ levels, narrow $\bar{B} D$ peaks could indicate nearly bound $b c \bar{q}_{k} \bar{q}_{l}$ tetraquark states [47].

\section{TERA-Z PROSPECTS}

In response to the discovery of the $125-\mathrm{GeV}$ Higgs boson, $H(125)$ [48], plans for large circular electronpositron colliders (FCC-ee [49] and CEPC [50]) are being developed as $e^{+} e^{-} \rightarrow H Z^{0}$ "Higgs factories" to run at c.m. energy $\sqrt{s} \approx 240 \mathrm{GeV}$. As now envisioned, these machines would have the added capability of high-luminosity running at $\sqrt{s}=M_{Z}$ that would accumulate $10^{12}$ examples of the reaction $e^{+} e^{-} \rightarrow Z^{0}$. With the observed branching fraction, $\mathcal{B}\left(Z^{0} \rightarrow b \bar{b}\right)=(15.12 \pm 0.05) \%$ [12], the tera$Z$ mode would produce some $3 \times 10^{11}$ boosted $b$-quarks, which would enable high-sensitivity searches for $(c \bar{b})$ states in a variety of decay channels. A recent computation suggests that $\mathcal{B}\left(Z^{0} \rightarrow(c \bar{b})+X\right) \approx 6 \times 10^{-4}[51]$.

The largest existing $e^{+} e^{-} \rightarrow Z^{0} \rightarrow$ hadrons datasets were recorded by experiments at CERN's Large Electron-Positron Collider during the 1990s. In samples of $(3.02,3.9$, and 4.2$)$ million hadronic $Z^{0}$ decays, the DELPHI, ALEPH, and OPAL Collaborations [52] found a small number of candidates for the decays $B_{c} \rightarrow J / \psi \pi^{+}$, $J / \psi \ell^{+} \nu$ and $J / \psi 3 \pi$. Those few specimens were not sufficient to establish a discovery, but the experiments were able to bound combinations of branching fractions $\mathcal{B}$ as

$\frac{\mathcal{B}\left(Z^{0} \rightarrow B_{c}+X\right)}{\mathcal{B}\left(Z^{0} \rightarrow \text { hadrons }\right)} \mathcal{B}\left(B_{c} \rightarrow\left\{\begin{array}{l}J / \psi \pi^{+} \\ J / \psi \ell^{+} \nu \\ J / \psi 3 \pi\end{array}\right\}\right) \lesssim$ few $\times 10^{-4}$,

at $90 \%$ confidence level, where $X$ denotes anything. The relative simplicity of $e^{+} e^{-} \rightarrow Z^{0}$ events and the boosted kinematics of resulting $B_{c}$ mesons suggest that a tera- $Z$ factory might be a felicitous choice to investigate $2 P \rightarrow$ $1 S+\gamma$ lines.

\section{CONCLUSIONS AND OUTLOOK}

In this article, we have presented a new analysis of the spectrum of mesons with beauty and charm. First, we modified the traditional Coulomb-plus-linear form of the quarkonium potential to incorporate running of the strong coupling constant $\alpha_{\mathrm{S}}$ that saturates at a fixed value at long distances. The new frozen- $\alpha_{\mathrm{s}}$ potential incorporates both perturbative and nonperturbative aspects of quantum chromodynamics. Second, we have set aside the perturbative treatment of spin splittings, instead incorporating lessons from lattice QCD and observations of the $(c \bar{c})$ and $(b \bar{b})$ spectra. 
We look forward to additional experimental progress, first by confirming and elaborating the characteristics of the $2 S$ levels reported by the CMS Collaboration [4]. Key observables are the mass of the $2^{1} S_{0}$ state, the splitting between the two lines, and the ratio of peak heights corrected for efficiencies and acceptance. It is also of interest to test whether the dipion mass spectra in the cascade decays $B_{c}^{\prime} \rightarrow B_{c} \pi^{+} \pi^{-}$and $B_{c}^{* \prime} \rightarrow B_{c}^{*} \pi^{+} \pi^{-}$ follow the pattern seen in $\psi(2 S) \rightarrow J / \psi \pi^{+} \pi^{-}$and $\Upsilon(2 S) \rightarrow \Upsilon(1 S) \pi^{+} \pi^{-}$decays. Although we expect the $3 S$ levels to lie above flavor threshold, exploring the $B_{c} \pi^{+} \pi^{-}$mass spectrum up through $7200 \mathrm{MeV}$ might yield indications of $3{ }^{1} S_{0} \rightarrow B_{c} \pi^{+} \pi^{-}$and $3{ }^{3} S_{1} \rightarrow$ $B_{c}^{*} \pi^{+} \pi^{-}$lines. The presence of one or the other of these could signal interactions of bound states with open channels. Prospecting for narrow $B^{(*)} D^{(*)}$ peaks near threshold could yield evidence of $B_{c}$ states beyond the $2 S$ levels.

The next frontier is the search for radiative transitions among $(c \bar{b})$ levels. The most promising candidate for first light is the $2^{3} P_{2}(6750) \rightarrow B_{c}^{*} \gamma$ transition. Determining the $B_{c}^{*}$ mass, perhaps by reconstructing $B_{c}^{*} \rightarrow B_{c} \gamma$, would provide an important check on lattice QCD calculations and a key input to future calculations.

Detecting the $B_{c} \rightarrow \tau \nu_{\tau}$ and $B_{c} \rightarrow p \bar{p} \pi^{+}$decays would be impressive experimental feats and would provide another test of the short-distance behavior of the groundstate wave function, complementing what will be learned from the $B_{c}^{*}-B_{c}$ splitting.

\section{ACKNOWLEDGMENTS}

This work was supported by Fermi Research Alliance, LLC under Award No. DE-AC02-07CH11359 with the U.S. Department of Energy, Office of Science, Office of High Energy Physics.

\section{APPENDIX: STRONG COUPLING EVOLUTION}

To make calculations with the frozen- $\alpha_{\mathrm{s}}$ potential, one must combine a linear term with a Coulomb term, $-4 \alpha_{\mathrm{s}}(r) / 3 r$, for which $\alpha_{\mathrm{s}}(r)$ is characterized by the solid red curve of Fig. 1. We present in Table IX numerical values of the strong coupling over the relevant range of distances, $0 \leq r \leq 0.8 \mathrm{fm}$. The entries advance in steps of $\delta \ln r=0.1$.
TABLE IX. Evolution of the strong coupling.

\begin{tabular}{|c|c|}
\hline$r(\mathrm{fm})$ & $\alpha_{\mathrm{s}}(r)$ \\
\hline 0.0080 & 0.1706 \\
\hline 0.0088 & 0.1742 \\
\hline 0.0097 & 0.1780 \\
\hline 0.0108 & 0.1819 \\
\hline 0.0119 & 0.1862 \\
\hline 0.0132 & 0.1908 \\
\hline 0.0145 & 0.1957 \\
\hline 0.0161 & 0.2007 \\
\hline 0.0178 & 0.2061 \\
\hline 0.0196 & 0.2116 \\
\hline 0.0217 & 0.2174 \\
\hline 0.0240 & 0.2235 \\
\hline 0.0265 & 0.2299 \\
\hline 0.0293 & 0.2365 \\
\hline 0.0323 & 0.2434 \\
\hline 0.0358 & 0.2505 \\
\hline 0.0395 & 0.2579 \\
\hline 0.0437 & 0.2659 \\
\hline 0.0483 & 0.2743 \\
\hline 0.0533 & 0.2829 \\
\hline 0.0589 & 0.2915 \\
\hline 0.0651 & 0.3001 \\
\hline 0.0720 & 0.3087 \\
\hline 0.0796 & 0.3171 \\
\hline 0.0879 & 0.3252 \\
\hline 0.0972 & 0.3330 \\
\hline 0.1074 & 0.3403 \\
\hline 0.1187 & 0.3471 \\
\hline 0.1312 & 0.3533 \\
\hline 0.1450 & 0.3590 \\
\hline 0.1602 & 0.3640 \\
\hline 0.1771 & 0.3685 \\
\hline 0.1957 & 0.3723 \\
\hline 0.2163 & 0.3757 \\
\hline 0.2390 & 0.3786 \\
\hline 0.2642 & 0.3811 \\
\hline 0.2920 & 0.3832 \\
\hline 0.3227 & 0.3849 \\
\hline 0.3566 & 0.3864 \\
\hline 0.3941 & 0.3876 \\
\hline 0.4355 & 0.3886 \\
\hline 0.4813 & 0.3895 \\
\hline 0.5320 & 0.3902 \\
\hline 0.5879 & 0.3908 \\
\hline 0.6497 & 0.3913 \\
\hline 0.7181 & 0.3917 \\
\hline 0.7936 & 0.3920 \\
\hline
\end{tabular}


[1] G. Aad et al. (ATLAS Collaboration), Observation of an Excited $B_{c}^{ \pm}$Meson State with the ATLAS Detector, Phys. Rev. Lett. 113, 212004 (2014).

[2] R. Aaij et al. (LHCb Collaboration), Search for excited $B_{c}^{+}$ states, J. High Energy Phys. 01 (2018) 138.

[3] For a recent assessment, see contributions to the Micro Workshop on $B_{c}^{+}$physics at LHCb, 13 July 2016, https://indico.cern.ch/event/549155/, especially Z. Yang, Experiment-Recent history of the $B_{c}^{+}$meson, https://indico.cern.ch/event/549155/contributions/2226387/ attachments/1308369/1957602/Bc_results20160713.pdf; A. Luchinsky, Theory-Status of the theoretical description of the $B_{c}^{+}$meson, https://indico.cern.ch/event/549155/ contributions/2226440/attachments/1308367/1956579/bc Luchinsky.pdf; R. Oldeman, Experiment-Perspectives for future studies, https://indico.cern.ch/event/549155/ contributions/2226443/attachments/1308858/1957460/ 20160713_Bc.pdf; A. V. Berezhnoy, Theory- $B_{c}^{+}$production and spectroscopy, https://indico.cern.ch/event/549155/ contributions/2226438/attachments/1309000/1957694/ Berezhnoy_Bc.pdf.

[4] A. M. Sirunyan et al. (CMS Collaboration), Observation of two excited $\mathrm{B}_{\mathrm{c}}^{+}$states and measurement of the $\mathrm{B}_{\mathrm{c}}^{+}(2 \mathrm{~S})$ mass in pp collisions at $\sqrt{s}=13 \mathrm{TeV}$, arXiv:1902.00571.

[5] E. Eichten and F. Feinberg, Spin dependent forces in QCD, Phys. Rev. D 23, 2724 (1981).

[6] E. J. Eichten and C. Quigg, Mesons with beauty and charm: Spectroscopy, Phys. Rev. D 49, 5845 (1994).

[7] For other work in a similar spirit, see V. V. Kiselev, A. K. Likhoded, and A. V. Tkabladze, $B_{c}$ spectroscopy, Phys. Rev. D 51, 3613 (1995); L. P. Fulcher, Phenomenological predictions of the properties of the $B_{c}$ system, Phys. Rev. D 60, 074006 (1999); S. Godfrey and N. Isgur, Mesons in a relativized quark model with chromodynamics, Phys. Rev. D 32, 189 (1985); S. Godfrey, Spectroscopy of $B_{c}$ mesons in the relativized quark model, Phys. Rev. D 70, 054017 (2004); D. Ebert, R. N. Faustov, and V. O. Galkin, Properties of heavy quarkonia and $B_{c}$ mesons in the relativistic quark model, Phys. Rev. D 67, 014027 (2003); A. V. Berezhnoy, V. V. Kiselev, A. K. Likhoded, and A. I. Onishchenko, $B_{c}$ meson at LHC, Yad. Fiz. 60N10, 1889 (1997) [Phys. At. Nucl. 60, 1729 (1997)]; N. R. Soni, B. R. Joshi, R. P. Shah, H. R. Chauhan, and J. N. Pandya, $Q \bar{Q}(Q \in\{b, c\})$ spectroscopy using the Cornell potential, Eur. Phys. J. C 78, 592 (2018).

[8] F. Abe et al. (CDF Collaboration), Observation of the $B_{c}$ Meson in $p \bar{p}$ Collisions at $\sqrt{s}=1.8 \mathrm{TeV}$, Phys. Rev. Lett. 81, 2432 (1998).

[9] T. Aaltonen et al. (CDF Collaboration), Observation of the Decay $B_{c}^{+} \rightarrow J / \psi \pi^{ \pm}$and Measurement of the $B_{c}^{+}$Mass, Phys. Rev. Lett. 100, 182002 (2008).

[10] V. M. Abazov et al. (D0 Collaboration), Observation of the $B_{c}$ Meson in the Exclusive Decay $B_{c} \rightarrow J / \psi \pi$, Phys. Rev. Lett. 101, 012001 (2008).

[11] R. Aaij et al. (LHCb Collaboration), Measurements of $B_{c}^{+}$ Production and Mass with the $B_{c}^{+} \rightarrow J / \psi \pi^{+}$Decay, Phys. Rev. Lett. 109, 232001 (2012); Observation of $B_{c}^{+} \rightarrow$ $J / \psi D_{s}^{+}$and $B_{c}^{+} \rightarrow J / \psi D_{s}^{*+}$ decays, Phys. Rev. D 87, 112012 (2013); Publisher's Note 89, 019901(A) (2014);
First Observation of a Baryonic $B_{c}^{+}$Decay, Phys. Rev. Lett. 113, 152003 (2014).

[12] M. Tanabashi et al. (Particle Data Group), Review of particle physics, Phys. Rev. D 98, 030001 (2018).

[13] I. F. Allison, C. T. H. Davies, A. Gray, A. S. Kronfeld, P. B. Mackenzie, and J. N. Simone (Fermilab Lattice, HPQCD, and UKQCD Collaborations), Mass of the $B_{c}$ Meson in Three-Flavor Lattice QCD, Phys. Rev. Lett. 94, 172001 (2005).

[14] R. J. Dowdall, C. T. H. Davies, T. C. Hammant, and R. R. Horgan, Precise heavy-light meson masses and hyperfine splittings from lattice QCD including charm quarks in the sea, Phys. Rev. D 86, 094510 (2012).

[15] We use spectroscopic notation $n^{2 S+1} L_{J}$, where $n$ is the principal quantum number, $S$ is total spin, and $L=$ $S, P, D, \ldots$ represents the angular momentum $0,1,2, \ldots$.

[16] M. Beneke and G. Buchalla, The $B_{c}$ meson lifetime, Phys. Rev. D 53, 4991 (1996); A. Yu. Anisimov, I. M. Narodetsky, C. Semay, and B. Silvestre-Brac, The $B_{c}$ meson lifetime in the light front constituent quark model, Phys. Lett. B 452, 129 (1999); V. V. Kiselev, A. E. Kovalsky, and A. K. Likhoded, $B_{c}$ decays and lifetime in QCD sum rules, Nucl. Phys. B585, 353 (2000); C.-H. Chang, S.-L. Chen, T.-F. Feng, and X.-Q. Li, The lifetime of $B_{c}$ meson and some relevant problems, Phys. Rev. D 64, 014003 (2001).

[17] N. Brambilla et al. (Quarkonium Working Group), Heavy quarkonium physics, CERN Yellow Report No. CERN2005-005, 2005, DOI: 10.5170/CERN-2005-005.

[18] A. Ali, L. Maiani, A. D. Polosa, and V. Riquer, $B_{c}^{ \pm}$decays into tetraquarks, Phys. Rev. D 94, 034036 (2016); A. Esposito, M. Papinutto, A. Pilloni, A. D. Polosa, and N. Tantalo, Doubly charmed tetraquarks in $B_{c}$ and $\Xi_{b c}$ decays, Phys. Rev. D 88, 054029 (2013); W. Wang, Y.-L. Shen, and C.-D. Lu, The study of $B_{c}^{-} \rightarrow X(3872) \pi^{-}\left(K^{-}\right)$decays in the covariant light-front approach, Eur. Phys. J. C 51, 841 (2007); W. Wang and Q. Zhao, Decipher the short-distance component of $X(3872)$ in $B_{c}$ decays, Phys. Lett. B 755, 261 (2016).

[19] A. Lytle, B. Colquhoun, C. Davies, and J. Koponen, $B_{c}$ spectroscopy using highly improved staggered quarks, Proc. Sci. LATTICE2018 (2018) 083.

[20] T. Appelquist and H. D. Politzer, Heavy Quarks and $e^{+} e^{-}$ Annihilation, Phys. Rev. Lett. 34, 43 (1975).

[21] T. Appelquist, A. De Rujula, H. D. Politzer, and S. L. Glashow, Spectroscopy of the New Mesons, Phys. Rev. Lett. 34, 365 (1975); E. Eichten, K. Gottfried, T. Kinoshita, J. B. Kogut, K. D. Lane, and T.-M. Yan, Spectrum of Charmed Quark-Antiquark Bound States, Phys. Rev. Lett. 34, 369 (1975); Erratum, Phys. Rev. Lett. 36, 1276(E) (1976).

[22] E. Eichten, K. Gottfried, T. Kinoshita, K. D. Lane, and T.-M. Yan, Charmonium: The Model, Phys. Rev. D 17, 3090 (1978); , Charmonium: The Model, Phys. Rev. D 21, 313(E) (1980); Charmonium: Comparison with experiment, Phys. Rev. D 21, 203 (1980).

[23] E. J. Eichten, K. Lane, and C. Quigg, Charmonium levels near threshold and the narrow state $X(3872) \rightarrow \pi^{+} \pi^{-} J / \psi$, Phys. Rev. D 69, 094019 (2004); New states above charm threshold, Phys. Rev. D 73, 014014 (2006); Erratum, Phys. Rev. D 73, 079903(E) (2006). 
[24] N. Brambilla et al., Heavy quarkonium: Progress, puzzles, and opportunities, Eur. Phys. J. C 71, 1534 (2011).

[25] Y. Sumino, Computation of heavy quarkonium spectrum in perturbative QCD, Proc. Sci. LL2016 (2016) 011.

[26] A. Martin, A fit of upsilon and charmonium spectra, Phys. Lett. 93B, 338 (1980).

[27] J. L. Richardson, The heavy quark potential and the $\Upsilon, J / \psi$ systems, Phys. Lett. 82B, 272 (1979).

[28] W. Buchmüller and S. H. H. Tye, Quarkonia and quantum chromodynamics, Phys. Rev. D 24, 132 (1981).

[29] V. N. Gribov, The theory of quark confinement, Eur. Phys. J. C 10, 91 (1999).

[30] K. G. Chetyrkin, Four-loop renormalization of QCD: Full set of renormalization constants and anomalous dimensions, Nucl. Phys. B710, 499 (2005); M. Czakon, The Four-loop QCD beta-function and anomalous dimensions, Nucl. Phys. B710, 485 (2005).

[31] M. Jeżabek, M. Peter, and Y. Sumino, On the relation between QCD potentials in momentum and position space, Phys. Lett. B 428, 352 (1998).

[32] D. Gromes, Spin dependent potentials in QCD and the correct long range spin orbit term, Z. Phys. C 26, 401 (1984).

[33] C. T. H. Davies, K. Hornbostel, G. P. Lepage, A. J. Lidsey, J. Shigemitsu, and J. H. Sloan, $B_{c}$ spectroscopy from lattice QCD, Phys. Lett. B 382, 131 (1996).

[34] E. B. Gregory, C. T. H. Davies, E. Follana, E. Gamiz, I. D. Kendall, G. P. Lepage, H. Na, J. Shigemitsu, and K. Y. Wong, A Prediction of the $B_{c}^{*}$ Mass in Full Lattice QCD, Phys. Rev. Lett. 104, 022001 (2010).

[35] N. Mathur, M. Padmanath, and S. Mondal, Precise Predictions of Charmed-Bottom Hadrons from Lattice QCD, Phys. Rev. Lett. 121, 202002 (2018).

[36] C. Quigg and J.L. Rosner, Counting narrow levels of quarkonium, Phys. Lett. 72B, 462 (1978).

[37] E. Eichten and K. Gottfried, Heavy quarks in $e^{+} e^{-}$ annihilation, Phys. Lett. 66B, 286 (1977).

[38] L. S. Brown and R. N. Cahn, Chiral Symmetry and $\psi^{\prime} \rightarrow$ $\psi \pi \pi$ Decay, Phys. Rev. Lett. 35, 1 (1975).

[39] See Sec. 7 of Ref. [17] and Sec. 3.3 of Ref. [24] for surveys of cascade decays.

[40] We adopt the conventional normalization, $\int d \Omega$ : $Y_{\ell m}^{*}(\theta, \phi) Y_{\ell^{\prime} m^{\prime}}(\theta, \phi)=\delta_{\ell \ell^{\prime}} \delta_{m m^{\prime}}$. See, e.g., the Appendix of H. A. Bethe and E. E. Salpeter, Quantum Mechanics of One- and Two-Electron Atoms (Springer-Verlag, Berlin, 1957).

[41] R. Van Royen and V. F. Weisskopf, Hadron decay processes and the quark model, Nuovo Cimento A 50, 617 (1967); Erratum, Nuovo Cimento A 51, 583(E) (1967). The factor of 3 accounts for quark color.

[42] E. Braaten and S. Fleming, QCD radiative corrections to the leptonic decay rate of the $B_{c}$ meson, Phys. Rev. D 52, 181 (1995); A. V. Berezhnoy, V. V. Kiselev, and A. K. Likhoded,
Photonic production of S- and $\mathrm{P}$ wave $B_{c}$ states and doubly heavy baryons, Z. Phys. A 356, 89 (1996).

[43] B. Colquhoun, C. T. H. Davies, R. J. Dowdall, J. Kettle, J. Koponen, G. P. Lepage, and A. T. Lytle (HPQCD Collaboration), B-meson decay constants: A more complete picture from full lattice QCD, Phys. Rev. D 91, 114509 (2015); For further work on semileptonic decays, see A. Lytle, B. Colquhoun, C. Davies, J. Koponen, and C. McNeile, Semileptonic $B_{c}$ decays from full lattice QCD, Proc. Sci., BEAUTY2016 (2016) 069; A. Lytle, Theory-Semileptonic $B_{c}^{+}$decays and Lattice QCD, in Ref. [3], https://indico .cern.ch/event/549155/contributions/2226442/attachments/ 1308725/1957203/Bc_lattice.pdf.

[44] M. J. Baker, J. Bordes, C. A. Dominguez, J. Penarrocha, and K. Schilcher, B meson decay constants $f_{B_{c}}, f_{B_{s}}$ and $f_{B}$ from QCD sum rules, J. High Energy Phys. 07 (2014) 032.

[45] C.-H. Chang, J.-X. Wang, and X.-G. Wu, BCVEGPY2.0: An upgraded version of the generator BCVEGPY with the addition of hadroproduction of the $P$-wave $B_{c}$ states, Comput. Phys. Commun. 174, 241 (2006). We use (derivatives of) wave functions at the origin derived from our current work. The quark mass parameters in this program vary with the produced state, to reproduce its mass. $1 S: m_{b}=5.000, m_{c}=1.275 ; 2 S: m_{b}=5.234, m_{c}=1.633$; $2 P: m_{b}=5.184, m_{c}=1.573 ; 3 S: m_{b}=5.447, m_{c}=1.825$; $3 P: m_{b}=5.502, m_{c}=1.633$, all in GeV.

[46] In an effective power-law potential $V(r)=\lambda r^{\nu}, \Delta_{21}<0$ so long as $\nu<1$. See Secs. 4.1.1 and 5.3.2 of C. Quigg and J.L. Rosner, Quantum mechanics with applications to quarkonium, Phys. Rep. 56, 167 (1979), particularly Eqs. (4.21) and (4.22).

[47] E. J. Eichten and C. Quigg, Heavy-Quark Symmetry Implies Stable Heavy Tetraquark Mesons $Q_{i} Q_{j} \bar{q}_{k} \bar{q}_{l}$, Phys. Rev. Lett. 119, 202002 (2017).

[48] G. Aad et al. (ATLAS Collaboration), Observation of a new particle in the search for the Standard Model Higgs boson with the ATLAS detector at the LHC, Phys. Lett. B 716, 1 (2012); S. Chatrchyan et al. (CMS Collaboration), Observation of a new boson at a mass of $125 \mathrm{GeV}$ with the CMS experiment at the LHC, Phys. Lett. B 716, 30 (2012).

[49] FCC-ee, the electron-positron option of the future circular colliders design study, http://tlep.web.cern.ch.

[50] CEPC, Circular electron-positron collider, http://cepc.ihep ac.cn.

[51] Q.-L. Liao, Y. Yu, Y. Deng, G.-Y. Xie, and G.-C. Wang, Excited heavy quarkonium production via $Z^{0}$ decays at a high luminosity collider, Phys. Rev. D 91, 114030 (2015).

[52] P. Abreu et al. (DELPHI Collaboration), Search for the $B_{c}$ meson, Phys. Lett. B 398, 207 (1997); R. Barate et al. (ALEPH Collaboration), Search for the $B_{c}$ meson in hadronic $Z$ decays, Phys. Lett. B 402, 213 (1997); K. Ackerstaff et al. (OPAL Collaboration), Search for the $B_{c}$ meson in hadronic $Z^{0}$ decays, Phys. Lett. B 420, 157 (1998). 